Chapter 31

\title{
Quantum Effects Through a Fractal Theory of Motion
}

\author{
M. Agop, C.Gh. Buzea, S. Bacaita, A. Stroe and M. Popa \\ Additional information is available at the end of the chapter \\ http://dx.doi.org/10.5772/54172
}

\section{Introduction}

Scale Relativity Theory (SRT) affirms that the laws of physics apply in all reference systems, whatever its state of motion and its scale. In consequence, SRT imply [1-3] the followings:

i. Particle movement on continuous and non-differentiable curve (or almost nowhere differentiable), that is explicitly scale dependent and its length tends to infinity, when the scale interval tends to zero.

ii. Physical quantities will be expressed through fractal functions, namely through functions that are dependent both on coordinate field and resolution scale. The invariance of the physical quantities in relation with the resolution scale generates special types of transformations, called resolution scale transformations. In what follows we will explain the above statement.

Let $F(x)$ be a fractal function in the interval $x \in[a, b]$ and let the sequence of values for $x$ be:

$$
x_{a}=x_{0}, x_{1}=x_{0}+\varepsilon, x_{k}=x_{0}+k \varepsilon, x_{n}=x_{0}+n \varepsilon=x_{b}
$$

We can now say that $F(x, \varepsilon)$ is a-scale approximation.

Let us now consider as a $\bar{\varepsilon}$-scale approximation of the same function. Since $F(x)$ is everywhere almost self-similar, if $\varepsilon$ and $\bar{\varepsilon}$ are sufficiently small, both approximations $F(x, \varepsilon)$ and must lead to same results. By comparing the two cases, one notices that scale expansion is related to the increase $d \varepsilon$ of $\varepsilon$, according to an increase $d \bar{\varepsilon}$ of $\bar{\varepsilon}$. But, in this case we have:

$$
\frac{d \varepsilon}{\varepsilon}=\frac{d \bar{\varepsilon}}{\bar{\varepsilon}}=d \rho
$$


situation in which we can consider the infinitesimal scale transformation as being

$$
\varepsilon^{\prime}=\varepsilon+d \varepsilon=\varepsilon+\varepsilon d \rho
$$

Such transformation in the case of function $F(x, \varepsilon)$, leads to:

$$
F\left(x, \varepsilon^{\prime}\right)=F(x, \varepsilon+\varepsilon d \rho)
$$

respectively, if we limit ourselves to a first order approximation:

$$
F\left(x, \varepsilon^{\prime}\right)=F(x, \varepsilon)+\frac{\partial F(x, \varepsilon)}{\partial \varepsilon}\left(\varepsilon^{\prime}-\varepsilon\right)=F(x, \varepsilon)+\frac{\partial F(x, \varepsilon)}{\partial \varepsilon} \varepsilon d \rho
$$

Moreover, let us notice that for an arbitrary but fixed $\varepsilon_{0}$, we obtain:

$$
\frac{\partial \ln \left(\varepsilon / \varepsilon_{0}\right)}{\partial \varepsilon}=\frac{\partial\left(\ln \varepsilon-\ln \varepsilon_{0}\right)}{\partial \varepsilon}=\frac{1}{\varepsilon}
$$

situation in which (5) can be written as:

$$
F\left(x, \varepsilon^{\prime}\right)=F(x, \varepsilon)+\frac{\partial F(x, \varepsilon)}{\partial \ln \left(\varepsilon / \varepsilon_{0}\right)} d \rho=\left[1+\frac{\partial}{\partial \ln \left(\varepsilon / \varepsilon_{0}\right)} d \rho\right] F(x, \varepsilon)
$$

Therefore, we can introduce the dilatation operator:

$$
\hat{D}=\frac{\partial}{\partial \ln \left(\varepsilon / \varepsilon_{0}\right)}
$$

At the same time, relation (8) shows that the intrinsic variable of resolution is not $\varepsilon$, but $\ln \left(\varepsilon / \varepsilon_{0}\right)$.

The fractal function is explicitly dependent on the resolution $\left(\varepsilon / \varepsilon_{0}\right)$, therefore we have to solve the differential equation:

$$
\frac{d F}{d \ln \left(\varepsilon / \varepsilon_{0}\right)}=P(F)
$$


where $P(F)$ is now an unknown function. The simplest explicit suggested form for $P(F)$ is linear dependence [2]

$$
P(F)=A+B F, A, B=\text { const }
$$

in which case the differential equation (9) takes the form:

$$
\frac{d F}{d \ln \left(\varepsilon / \varepsilon_{0}\right)}=A+B F
$$

Hence by integration and substituting:

$$
\begin{gathered}
B=-\tau, \\
-\frac{A}{B}=F_{0}
\end{gathered}
$$

we obtain:

$$
F\left(\frac{\varepsilon}{\varepsilon_{0}}\right)=F_{0}\left[1+\left(\frac{\varepsilon_{0}}{\varepsilon}\right)^{\tau}\right]
$$

We can now generalize the previous result by considering that $F$ is dependent on parameterization of the fractal curve. If $p$ characterizes the position on the fractal curve then, following the same algorithm as above, the solution will be as a sum of two terms i.e. both classical and differentiable (depending only on position) and fractal, non-differentiable (depending on position and, divergently, on $\left.\varepsilon / \varepsilon_{0}\right)$

$$
F\left(p, \varepsilon / \varepsilon_{0}\right)=F_{0}(p)\left[1+\xi(p)\left(\frac{\varepsilon_{0}}{\varepsilon}\right)^{\tau(p)}\right]
$$

where $\xi(p)$ is a function depending on parameterization of the fractal curve.

The following particular cases are to be considered:

1. in asymptotic small scale regime $\varepsilon\left\langle\left\langle\varepsilon_{0}, \tau\right.\right.$ is constant (with no scale dependence) and power-law dependence on resolution is obtained: 


$$
\begin{array}{ll}
F\left(p, \varepsilon / \varepsilon_{0}\right)=T(p)\left(\frac{\varepsilon_{0}}{\varepsilon}\right)^{\tau} & \mathrm{a} \\
T(p)=F_{0}(p) Q(p) & \mathrm{b}
\end{array}
$$

2. in the asymptotic big scale regime $\varepsilon\rangle\rangle \varepsilon_{0}$, $\tau$ is constant (with no scale dependence) and, in terms of resolution, one obtains an independent law:

$$
F\left(p, \varepsilon / \varepsilon_{0}\right) \rightarrow F_{0}(p)
$$

Particularly, if $F\left(p, \varepsilon / \varepsilon_{0}\right)$ are the coordinates in given space, we can write

$$
X\left(p, \varepsilon / \varepsilon_{0}\right)=x(p)\left[1+\xi(p)\left(\frac{\varepsilon_{0}}{\varepsilon}\right)^{\tau}\right]
$$

In this situation, $\xi(p)$ becomes a highly fluctuating function which can be described by stochastic process while $\tau$ represents (according to previous description) the difference between fractal and topological dimensions. The result is a sum of two terms, a classical, differentiable one (dependent only on the position) and a fractal, non-differentiable one (dependent both on the position and, divergently, on $\varepsilon / \varepsilon_{0}$ ). This represents the importance of the above analysis.

By differentiating these two parts we obtain:

$$
d X=d x+d \xi
$$

where $d x$ is the classical differential element and $d \xi$ is a differential fractal one.

iii. There is infinity of fractal curves (geodesics) relating to any couple of points (or starting from any point) and applied for any scale. The phenomenon can be easily understood at the level of fractal surfaces, which, in their turn, can be described in terms of fractal distribution of conic points of positive and negative infinite curvature. As a consequence, we have replaced velocity on a particular geodesic by fractal velocity field of the whole infinite ensemble of geodesics. This representation is similar to that of fluid mechanics [4] where the motion of the fluid is described in terms of its velocity field $v=(x(t), t)$, density $\rho=(x(t), t)$ and, possibly, its pressure. We shall, indeed, recover the fundamental equations of fluid mechanics (Euler and continuity equations), but we shall write them in terms of a density of probability (as defined by the set of geodesics) instead of a density of matter and adding an additional term of quantum pressure (the expression of fractal geometry). 
iv. The local differential time invariance is broken, so the time-derivative of the fractal field $Q$ can be written two-fold:

$$
\begin{array}{ll}
\frac{d_{+} Q}{d t}=\lim _{\Delta t \rightarrow 0_{+}} \frac{Q(t+\Delta t)-Q(t)}{\Delta t} & \mathrm{a} \\
\frac{d_{-} Q}{d t}=\lim _{\Delta t \rightarrow 0_{-}} \frac{Q(t)-Q(t-\Delta t)}{\Delta t} & \mathrm{~b}
\end{array}
$$

Both definitions are equivalent in the differentiable case $d t \rightarrow-d t$. In the non-differentiable situation, these definitions are no longer valid, since limits are not defined anymore. Fractal theory defines physics in relationship with the function behavior during the "zoom" operation on the time resolution $\delta t$, here identified with the differential element $d t$ (substitution principle), which is considered an independent variable. The standard field $Q(t)$ is therefore replaced by fractal field $Q(t, d t)$, explicitly dependent on time resolution interval, whose derivative is not defined at the unnoticeable limit $d t \rightarrow 0$. As a consequence, this leads to the two derivatives of the fractal field $Q$ as explicit functions of the two variables $t$ and $d t$,

$$
\begin{array}{ll}
\frac{d_{+} Q}{d t}=\lim _{\Delta t \rightarrow 0_{+}} \frac{Q(t+\Delta t, \Delta t)-Q(t, \Delta t)}{\Delta t} & \mathrm{a} \\
\frac{d_{-} Q}{d t}=\lim _{\Delta t \rightarrow 0_{-}} \frac{Q(t, \Delta t)-Q(t-\Delta t, \Delta t)}{\Delta t} & \mathrm{~b}
\end{array}
$$

Notation "+" corresponds to the forward process, while "-" to the backward one.

v. We denote the average of these vectors by $d x_{ \pm}^{i}$, i.e.

$$
\left\langle d X_{ \pm}^{i}\right\rangle=d x_{ \pm}^{i}, i=1,2
$$

Since, according to (19), we can write:

$$
d X_{ \pm}^{i}=d x_{ \pm}^{i}+d \xi_{ \pm}^{i}
$$

and it results:

$$
\left\langle d \xi_{ \pm}^{i}\right\rangle=0
$$

vi. The differential fractal part satisfies the fractal equation: 


$$
d_{ \pm} \xi^{i}=\lambda_{ \pm}^{i}(d t)^{1 / D_{F}}
$$

where $\lambda_{ \pm}^{i}$ are some constant coefficients and $D_{F}$ is a constant fractal dimension. We note that the use of any Kolmogorov or Hausdorff [1, 5, 6-8] definitions can be accepted for fractal dimension, but once a certain definition is admitted, it should be used until the end of analyzed dynamics.

vii. The local differential time reflection invariance is recovered by combining the two derivatives, $d_{+} / d t$ and $d_{-} / d t$, in the complex operator:

$$
\frac{\hat{d}}{d t}=\frac{1}{2}\left(\frac{d_{+}+d_{-}}{d t}\right)-\frac{i}{2}\left(\frac{d_{+}-d_{-}}{d t}\right)
$$

Applying this operator to the "position vector", a complex velocity yields

$$
\hat{V}=\frac{\hat{d} \boldsymbol{X}}{d t}=\frac{1}{2}\left(\frac{d_{+} X+d_{-} X}{d t}\right)-\frac{i}{2}\left(\frac{d_{+} X-d_{-} X}{d t}\right)=\frac{V_{+}+V_{-}}{2}-i \frac{V_{+}-V_{-}}{2}=V-i U
$$

with:

$$
\begin{array}{ll}
V=\frac{V_{+}+V_{-}}{2} & \mathrm{a} \\
U=\frac{V_{+}-V_{-}}{2} & \mathrm{~b}
\end{array}
$$

The real part, $V$, of the complex velocity $\hat{V}$, represents the standard classical velocity, which does not depend on resolution, while the imaginary part, $U$, is a new quantity coming from resolution dependant fractal.

\section{Covariant total derivative}

Let us now assume that curves describing particle movement (continuous but non-differentiable) are immersed in a 3-dimensional space, and that $X$ of components $X^{i}(i=\overline{1,3})$ is the position vector of a point on the curve. Let us also consider a fractal field $Q(X, t)$ and expand its total differential up to the third order:

$$
d_{ \pm} Q=\frac{\partial Q}{\partial t} d t+\nabla Q \cdot d_{ \pm} \mathbf{X}++\frac{1}{2} \frac{\partial^{2} Q}{\partial X^{i} \partial X^{j}} d_{ \pm} X^{i} d_{ \pm} X^{j}+\frac{1}{6} \frac{\partial^{3} Q}{\partial X^{i} \partial X^{j} \partial X^{k}} d_{ \pm} X^{i} d_{ \pm} X^{j} d_{ \pm} X^{k}
$$


where only the first three terms were used in Nottale's theory (i.e. second order terms in the motion equation). Relations (29) are valid in any point both for the spatial manifold and for the points $X$ on the fractal curve (selected in relations 29). Hence, the forward and backward average values of these relations take the form:

$$
\left\langle d_{ \pm} Q\right\rangle=\left\langle\frac{\partial Q}{\partial t} d t\right\rangle+\left\langle\nabla Q \cdot d_{ \pm} X\right\rangle+\frac{1}{2}\left\langle\frac{\partial^{2} Q}{\partial X^{i} \partial X^{j}} d_{ \pm} X^{i} d_{ \pm} X^{j}\right\rangle+\frac{1}{6}\left\langle\frac{\partial^{3} Q}{\partial X^{i} \partial X^{j} \partial X^{k}} d_{ \pm} X^{i} d_{ \pm} X^{j} d_{ \pm} X^{k}\right\rangle
$$

The following aspects should be mentioned: the mean value of function $f$ and its derivatives coincide with themselves and the differentials $d_{ \pm} X^{i}$ and $d t$ are independent; therefore, the average of their products coincides with the product of averages. Consequently, the equations (30) become:

$$
d_{ \pm} Q=\frac{\partial Q}{\partial t} d t+\nabla Q\left\langle d_{ \pm} X\right\rangle+\frac{1}{2} \frac{\partial^{2} Q}{\partial X^{i} \partial X^{j}}\left\langle d_{ \pm} X^{i} d_{ \pm} X^{j}\right\rangle+\frac{1}{6} \frac{\partial^{3} Q}{\partial X^{i} \partial X^{j} \partial X^{k}}\left\langle d_{ \pm} X^{i} d_{ \pm} X^{j} d_{ \pm} X^{k}\right\rangle
$$

or more, using equations (23) with characteristics (24),

$$
\begin{aligned}
& d_{ \pm} Q=\frac{\partial Q}{\partial t} d t+\nabla Q \cdot d_{ \pm} X+\frac{1}{2} \frac{\partial^{2} Q}{\partial X^{i} \partial X^{j}}\left(d_{ \pm} x^{i} d_{ \pm} x^{j}+\left\langle d_{ \pm} \xi^{i} d_{ \pm} \xi^{j}\right\rangle\right)+ \\
& \frac{1}{6} \frac{\partial^{3} Q}{\partial X^{i} \partial X^{j} \partial X^{k}}\left(d_{ \pm} x^{i} d_{ \pm} x^{j} d_{ \pm} x^{k}+\left\langle d_{ \pm} \xi^{i} d_{ \pm} \xi^{j} d_{ \pm} \xi^{k}\right\rangle\right)
\end{aligned}
$$

Even if the average value of the fractal coordinate $d_{ \pm} \xi^{i}$ is null (see 24), for higher order of fractal coordinate average, the situation can still be different. Firstly, let us focus on the averages $\left\langle d_{+} \xi^{i} d_{+} \xi^{j}\right\rangle$ and $\left\langle d_{-} \xi^{i} d_{-} \xi^{j}\right\rangle$. If $i \neq j$, these averages are zero due to the independence of $d_{ \pm} \xi^{i}$ and $d_{ \pm} \xi^{j}$. So, using (25), we can write:

$$
\left\langle d_{ \pm} \xi^{i} d_{ \pm} \xi^{j}\right\rangle=\lambda_{ \pm}^{i} \lambda_{ \pm}^{j}(d t)^{\left(2 / D_{F}\right)-1} d t
$$

Then, let us consider the averages $\left\langle d_{ \pm} \xi^{i} d_{ \pm} \xi^{j} d_{ \pm} \xi^{k}\right\rangle$. If $i \neq j \neq k$, these averages are zero due to independence of $d_{ \pm} \xi^{i}$ on $d_{ \pm} \xi^{j}$ and $d_{ \pm} \xi^{k}$. Now, using equations (25), we can write:

$$
\left\langle d_{ \pm} \xi^{i} d_{ \pm} \xi^{j} d_{ \pm} \xi^{k}\right\rangle=\lambda_{ \pm}^{i} \lambda_{+}^{j} \lambda_{ \pm}^{k}(d t)^{\left(3 / D_{F}\right)-1} d t
$$

Then, equations (32) may be written as follows: 


$$
\begin{aligned}
& d_{ \pm} Q=\frac{\partial Q}{\partial t} d t+d_{ \pm} x \cdot \nabla Q+\frac{1}{2} \frac{\partial^{2} Q}{\partial X^{i} \partial X^{j}} d_{ \pm} x^{i} d_{ \pm} x^{j}+\frac{1}{2} \frac{\partial^{2} Q}{\partial X^{i} \partial X^{j}} \lambda_{ \pm}^{i} \lambda_{ \pm}^{j}(d t)^{\left(2 / D_{F}\right)-1} d t+ \\
& \frac{1}{6} \frac{\partial^{3} Q}{\partial X^{i} \partial X^{j} \partial X^{k}} d_{ \pm} x^{i} d_{ \pm} x^{j} d_{ \pm} x^{k}+\frac{1}{6} \frac{\partial^{3} Q}{\partial X^{i} \partial X^{j} \partial X^{k}} \lambda_{ \pm}^{i} \lambda_{ \pm}^{j} \lambda_{ \pm}^{k}(d t)^{\left(3 / D_{F}\right)-1} d t
\end{aligned}
$$

If we divide by $d t$ and neglect the terms containing differential factors (for details on the method see $[9,10])$, equations (38a) and $(38 b)$ are reduced to:

$$
\frac{d_{ \pm} Q}{d t}=\frac{\partial Q}{\partial t}+V_{ \pm} \cdot \nabla Q+\frac{1}{2} \frac{\partial^{2} Q}{\partial X^{i} \partial X^{j}} \lambda_{ \pm}^{i} \lambda_{ \pm}^{j}(d t)^{\left(2 / D_{F}\right)-1}+\frac{1}{6} \frac{\partial^{3} Q}{\partial X^{i} \partial X^{j} \partial X^{k}} \lambda_{ \pm}^{i} \lambda_{ \pm}^{j} \lambda_{ \pm}^{k}(d t)^{\left(3 / D_{F}\right)-1}
$$

These relations also allow us to define the operator:

$$
\frac{d_{ \pm}}{d t}=\frac{\partial}{\partial t}+\boldsymbol{V}_{ \pm} \cdot \nabla+\frac{1}{2} \frac{\partial^{2}}{\partial X^{i} \partial X^{j}} \lambda_{ \pm}^{i} \lambda_{ \pm}^{j}(d t)^{\left(2 / D_{F}\right)-1}+\frac{1}{6} \frac{\partial^{3}}{\partial X^{i} \partial X^{j} \partial X^{k}} \lambda_{ \pm}^{i} \lambda_{ \pm}^{j} \lambda_{ \pm}^{k}(d t)^{\left(3 / D_{F}\right)-1}
$$

Under these circumstances, let us calculate $(\hat{\partial} Q / \partial t)$. Taking into account equations (26), (27) and (37), we shall obtain:

$$
\begin{aligned}
& \frac{\partial Q}{\partial t}=\frac{1}{2}\left[\frac{d_{+} Q}{d t}+\frac{d_{-} Q}{d t}-i\left(\frac{d_{+} Q}{d t}-\frac{d_{-} Q}{d t}\right)\right]= \\
& =\frac{1}{2} \frac{\partial Q}{\partial t}+\frac{1}{2} \boldsymbol{V}_{+} \cdot \nabla Q+\lambda_{+}^{i} \lambda_{+}^{j} \frac{1}{4}(d t)^{\left(2 / D_{F}\right)-1} \frac{\partial^{2} Q}{\partial X^{i} \partial X^{j}}+\lambda_{+}^{i} \lambda_{+}^{j} \lambda_{+}^{k} \frac{1}{12}(d t)^{\left(3 / D_{F}\right)-1} \frac{\partial^{3} Q}{\partial X^{i} \partial X^{j} \partial X^{k}}+ \\
& +\frac{1}{2} \frac{\partial Q}{\partial t}+\frac{1}{2} \boldsymbol{V}_{-} \cdot \nabla Q++\lambda_{-}^{i} \lambda_{-}^{j} \frac{1}{4}(d t)^{\left(2 / D_{F}\right)-1} \frac{\partial^{2} Q}{\partial X^{i} \partial X^{j}}+\lambda_{-}^{i} \lambda_{-}^{j} \lambda_{-}^{k} \frac{1}{12}(d t)^{\left(3 / D_{F}\right)-1} \frac{\partial^{3} Q}{\partial X^{i} \partial X^{j} \partial X^{k}}- \\
& -\frac{i}{2} \frac{\partial Q}{\partial t}-\frac{i}{2} \boldsymbol{V}_{+} \cdot \nabla Q-\lambda_{+}^{i} \lambda_{+}^{j} \frac{i}{2}(d t)^{\left(2 / D_{F}\right)-1} \frac{\partial^{2} Q}{\partial X^{i} \partial X^{j}}-\lambda_{+}^{i} \lambda_{+}^{j} \lambda_{+}^{k} \frac{i}{12}(d t)^{\left(3 / D_{F}\right)-1} \frac{\partial^{3} Q}{\partial X^{i} \partial X^{j} \partial X^{k}}+ \\
& +\frac{i}{2} \frac{\partial Q}{\partial t}+\frac{i}{2} \boldsymbol{V}_{-} \cdot \nabla Q+\lambda_{-}^{i} \lambda_{-}^{j} \frac{i}{2}(d t)^{\left(2 / D_{F}\right)-1} \frac{\partial^{2} Q}{\partial X^{i} \partial X^{j}}+\lambda_{-}^{i} \lambda_{-}^{j} \lambda_{-}^{k} \frac{i}{12}(d t)^{\left(3 / D_{F}\right)-1} \frac{\partial^{3} Q}{\partial X^{i} \partial X^{j} \partial X^{k}}= \\
& =\frac{\partial Q}{\partial t}+\left(\frac{\boldsymbol{V}_{+}+\boldsymbol{V}_{-}}{2}-i \frac{\boldsymbol{V}_{+}-\boldsymbol{V}_{-}}{2}\right) \cdot \nabla Q+\frac{(d t)^{\left(2 / D_{F}\right)-1}}{4}\left[\left(\lambda_{+}^{i} \lambda_{+}^{j}+\lambda_{-}^{i} \lambda_{-}^{j}\right)-i\left(\lambda_{+}^{i} \lambda_{+}^{j}-\lambda_{-}^{i} \lambda_{-}^{j}\right)\right] \frac{\partial^{2} Q}{\partial X^{i} \partial X^{j}}+ \\
& +\frac{(d t)^{\left(3 / D_{F}\right)-1}}{12}\left[\left(\lambda_{+}^{i} \lambda_{+}^{j} \lambda_{+}^{k}+\lambda_{-}^{i} \lambda_{-}^{j} \lambda_{-}^{k}\right)-i\left(\lambda_{+}^{i} \lambda_{+}^{j} \lambda_{+}^{k}-\lambda_{-}^{i} \lambda_{-}^{j} \lambda_{-}^{k}\right)\right] \frac{\partial^{3} Q}{\partial X^{i} \partial X^{j} \partial X^{k}}= \\
& =\frac{\partial Q}{\partial t}+\hat{\boldsymbol{V} \cdot \nabla Q} \cdot \frac{(d t)^{\left(2 / D_{F}\right)-1}}{4}\left[\left(\lambda_{+}^{i} \lambda_{+}^{j}+\lambda_{-}^{i} \lambda_{-}^{j}\right)-i\left(\lambda_{+}^{i} \lambda_{+}^{j}-\lambda_{-}^{i} \lambda_{-}^{j}\right)\right] \frac{\partial^{2} Q}{\partial X^{i} \partial X^{j}}+ \\
& \frac{(d t)^{\left(3 / D_{F}\right)-1}}{12}\left[\left(\lambda_{+}^{i} \lambda_{+}^{j} \lambda_{+}^{k}+\lambda_{-}^{i} \lambda_{-}^{j} \lambda_{-}^{k}\right)-i\left(\lambda_{+}^{i} \lambda_{+}^{j} \lambda_{+}^{k}-\lambda_{-}^{i} \lambda_{-}^{j} \lambda_{-}^{k}\right)\right] \frac{\partial^{3} Q}{\partial X^{i} \partial X^{j} \partial X^{k}}
\end{aligned}
$$


This relation also allows us to define the fractal operator:

$$
\begin{aligned}
& \frac{\hat{\partial}}{\partial t}=\frac{\partial}{\partial t}+\hat{V} \cdot \nabla+\frac{(d t)^{\left(2 / D_{F}\right)-1}}{4}\left[\left(\lambda_{+}^{i} \lambda_{+}^{j}+\lambda_{-}^{i} \lambda_{-}^{j}\right)-i\left(\lambda_{+}^{i} \lambda_{+}^{j}-\lambda_{-}^{i} \lambda_{-}^{j}\right)\right] \frac{\partial^{2}}{\partial X^{i} \partial X^{j}}+ \\
& +\frac{(d t)^{\left(3 / D_{F}\right)-1}}{12}\left[\left(\lambda_{+}^{i} \lambda_{+}^{j} \lambda_{+}^{k}+\lambda_{-}^{i} \lambda_{-}^{j} \lambda_{-}^{k}\right)-i\left(\lambda_{+}^{i} \lambda_{+}^{j} \lambda_{+}^{k}-\lambda_{-}^{i} \lambda_{-}^{j} \lambda_{-}^{k}\right)\right] \frac{\partial^{3}}{\partial X^{i} \partial X^{j} \partial X^{k}}
\end{aligned}
$$

Particularly, by choosing:

$$
\begin{gathered}
\lambda_{+}^{i} \lambda_{+}^{j}=-\lambda_{-}^{i} \lambda_{-}^{j}=2 \mathrm{D} \delta^{\mathrm{ij}} \\
\lambda_{+}^{i} \lambda_{+}^{j} \lambda_{+}^{k}=-\lambda_{-}^{i} \lambda_{-}^{j} \lambda_{+}^{k}=2 \sqrt{2} \mathrm{D}^{3 / 2} \delta^{\mathrm{jkk}}
\end{gathered}
$$

the fractal operator (39) takes the usual form:

$$
\frac{\hat{\partial}}{\partial t}=\frac{\partial}{\partial t}+\hat{\boldsymbol{V}} \cdot \nabla-i \mathbf{D}(d t)^{\left(2 / D_{F}\right)-1} \Delta+\frac{\sqrt{2}}{3} \mathbf{D}^{3 / 2}(d t)^{\left(3 / D_{F}\right)-1} \nabla^{3}
$$

We now apply the principle of scale covariance and postulate that the passage from classical (differentiable) to "fractal" mechanics can be implemented by replacing the standard time derivative operator, $d / d t$, with the complex operator $\partial / \partial t$ (this results in a generalization of Nottale's [1,2] principle of scale covariance). Consequently, we are now able to write the diffusion equation in its covariant form:

$$
\frac{\partial}{\partial t}=\frac{\partial Q}{\partial t}+(\hat{\boldsymbol{V}} \cdot \nabla) Q-i D(d t)^{\left(2 / D_{F}\right)-1} \Delta Q+\frac{\sqrt{2}}{3} D^{3 / 2}(d t)^{\left(3 / D_{F}\right)-1} \nabla^{3} Q=0
$$

This means that at any point on a fractal path, the local temporal $\partial_{t} Q$, the non-linear (convective), $(\hat{V} \cdot \nabla) Q$, the dissipative, $\Delta Q$, and the dispersive, $\nabla^{3} Q$, terms keep their balance.

\section{Fractal space-time and the motion equation of free particles in the dissipative approximation}

Newton's fundamental equation of dynamics in the dissipative approximation is: 


$$
m \frac{\hat{\partial} \hat{V}}{\partial t}=-\nabla \Phi
$$

where $m$ is the mass, $\hat{V}$ the instantaneous velocity of the particle, $\Phi$ the scalar potential and

$$
\frac{\hat{\partial}}{\partial t}=\frac{\partial}{\partial t}+(\hat{\boldsymbol{V}} \cdot \nabla)-i D(d t)^{\left(2 / D_{F}\right)-1} \Delta
$$

is the fractal operator in the dissipative approximation.

In what follows, we study what happens with equation (44), in the free particle case $(\Phi=0)$, if one considers the space-time where particles move changes from classical to nondifferentiable.

According to Nottale [11], the transition from classical (differentiable) mechanics to the scale relativistic framework is implemented by passing to a fluid-like description (the fractality of space), considering the velocity field a fractal function explicitly depending on a scale variable (the fractal geometry of each geodesic). Separating the real and imaginary parts, (44) becomes:

$$
\begin{aligned}
& \frac{\partial \mathbf{V}}{\partial t}+\mathbf{V} \cdot \nabla \mathbf{V}=0 \\
& \mathbf{U} \cdot \nabla \mathbf{V}=-\mathrm{D} \Delta \mathbf{V}
\end{aligned}
$$

where $\hat{V}=V-i \boldsymbol{U}$ is the complex velocity defined through (27) and $D$ defines the amplitude of the fractal fluctuations $\left(\mathrm{D}=D(d t)^{\left(2 / D_{F}\right)-1}\right)$.

Let us analyze in what follows, the second equation (46) which, one can see, may contain some interesting physics. If we compare it with Navier-Stokes equation, from fluid mechanics [12]

$$
\frac{D \boldsymbol{v}}{D t}=\frac{\partial \boldsymbol{v}}{\partial t}+\boldsymbol{v} \cdot \nabla \boldsymbol{v}=v \nabla^{2} \boldsymbol{v}
$$

we can see the left side of (46) gives the rate at which $V$ is transported through a 'fluid' by means of the motion of 'fluid' particles with the velocity $U$; the right hand side gives the diffusion of $V,(D$ which is the amplitude of the fractal fluctuations, plays here the role of the 'cinematic viscosity' of the 'fluid'). One can notice, in those regions in which the right hand side of (47) is negligible, $D v / D t=0$. This means that in inviscid flows, for instance, $\hat{V}$ is frozen into the 'particles of the fluid'. Physically this is due to the fact that in an inviscid 'fluid' shear stresses are zero, so that there is no mechanism by which $\hat{V}$ can be transferred from one 'fluid' particle to another. This may be the case for the transport of $V$ by $U$ in the second equation (II.3). 
If we consider the flow of $V$ induced by a uniform translational motion of a plane spaced a distance $Y$ above a stationary parallel plane (Fig. 1), and if the 'fluid' velocity increases from zero (at the stationary plane) to $U$ (at the moving plane) like in the case of simple Couette flow, or simple shear flow, then

$$
\text { rate of shear deformation }=\frac{d V}{d y}=\frac{U}{Y}
$$

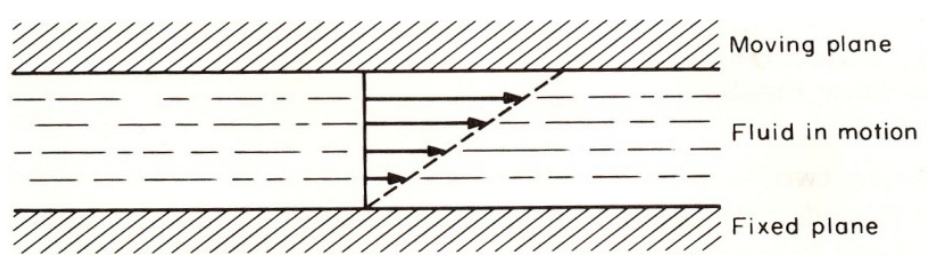

Figure 1. Uniform translational motion of a plane spaced a distance $Y$ above a stationary parallel plane.

For many fluids it is found that the magnitude of the shearing stress is related to the rate of shear proportionally:

$$
T=\eta \frac{d V}{d y}=\eta \frac{U}{Y}
$$

Fluids which obey (49) in the above situation are known as Newtonian fluids, which have a very small coefficient of viscosity. When such 'fluids' flow at reasonable velocities it is found that viscous effects appear only in thin layers on the surface of objects or surfaces over which the 'fluid' flows. That is, if one continues the analogy, and questions how is $V$ transported by the motion of 'fluid' particles with the velocity $U$, in second equation (46), one can assume that the mechanism of transfer of $V$ from one particle of 'fluid' to another is achieved over small distances (in thin layers, as stated above).

We study an important case, of the one-dimensional flow along the Ox axis :

$$
\boldsymbol{V}=\zeta(x) \boldsymbol{k}
$$

To resume, the model considered here consists in analyzing the transport of $V$, along a small elementary distance $\Lambda$, by the 'particles' of a Newtonian fluid moving with velocity $\boldsymbol{U}$, where the stress tensor obeys (49), i.e. 


$$
\frac{d \zeta(x)}{d x}=\frac{\zeta(x)}{\Lambda}
$$

like in the case of simple Couette flow, or simple shear flow.

Consequently, the second eq. (46) reduces to the scalar equation

$$
\zeta^{\prime \prime}(x)+K^{2}(x) \zeta(x)=0
$$

which is the time independent Schrödinger equation, and

$$
K^{2}(x)=\frac{1}{\Lambda D} U(x)
$$

with $\Lambda$ and $D$ having the significance of a small elementary distance and of the 'cinematic viscosity' (or amplitude of the fractal fluctuations), respectively, and $U(x)$ is the velocity of the 'Newtonian fluid', which is nothing but the imaginary part of the complex velocity [13]. In what follows, we solve this equation accurately by means of the WKBJ approximation method with connection formulas.

\subsection{Solving the Schrödinger type equation by means of the WKBJ approximation method}

Let us re-write (53) in the form

$$
K^{2}(x)=\frac{1}{\Lambda D} U(x)=\frac{2 m^{2} c}{\hbar^{2}}(\chi-\gamma(x))
$$

where we take $D=\hbar / 2 m$ and consider the small elementary distance the Compton length $\Lambda=\hbar / m c$ [14]. Therefore, the Schrödinger equation (52) splits into:

$$
\frac{d^{2}}{d x^{2}} \zeta(x)+k^{2}(x) \zeta(x)=0, \quad \chi>\gamma \text { or } \frac{d^{2}}{d x^{2}} \zeta(x)-\rho^{2}(x) \zeta(x)=0, \quad \chi<\gamma
$$

where

$$
k(x)=\sqrt{\frac{2 \mu(\chi-\gamma(x))}{\hbar^{2}}}, \rho(x)=\sqrt{\frac{2 \mu(\gamma(x)-\chi)}{\hbar^{2}}} \text { with } \mu=m^{2} c
$$

$\chi$ is a limit velocity and $\gamma(x)$ a 'velocity potential'. 
Let us try a solution of the form $\zeta(x)=A \exp ((i / \hbar) S(x))$. Substituting this solution into the timeindependent Schrödinger equation (52) we get:

$$
i \hbar \frac{d^{2} S}{d x^{2}}-\left(\frac{d S}{d x}\right)^{2}+\hbar^{2} k^{2}=0 \text { or } i \hbar \frac{d^{2} S}{d x^{2}}-\left(\frac{d S}{d x}\right)^{2}-\hbar^{2} \rho^{2}=0
$$

Assume that $\hbar$ can, in some sense, be regarded as a small quantity and that $S(x)$ can be expanded in powers of $\hbar, S(x)=S_{0}(x)+\hbar S_{1}(x)+\ldots$.

Then,

$$
i \hbar \frac{d}{d x}\left[\frac{d S_{0}}{d x}+\hbar \frac{d S_{1}}{d x}+\ldots\right]-\left(\frac{d S_{0}}{d x}+\hbar \frac{d S_{1}}{d x}+\ldots\right)^{2}+\hbar^{2} k^{2}=0, \quad(\chi>\gamma(x))
$$

We assume that $\left|\frac{d S_{0}}{d x}\right|>>\left|\hbar \frac{d S_{1}}{d x}\right|$ and collect terms with equal powers of $\hbar$.

$$
\begin{aligned}
& -\left[\frac{d S_{0}}{d x}\right]^{2}+\hbar^{2} k^{2}=0 \Rightarrow S_{0}= \pm \int_{x} \hbar k\left(x^{\prime}\right) d x^{\prime} \\
& i \frac{d^{2} S_{0}}{d x^{2}}-2 \frac{d S_{0}}{d x} \frac{d S_{1}}{d x}=0 \Rightarrow S_{1}=\frac{1}{2} i \ln k(x)
\end{aligned}
$$

We have used:

$$
i \frac{d}{d x}\left(\frac{d S_{0}}{d x}\right)=2 \frac{d S_{0}}{d x} \frac{d S_{1}}{d x}, \quad \mathrm{i} \frac{\mathrm{dk}}{\mathrm{dx}}=2 k \frac{d S_{1}}{d x}, \quad d S_{1}=\frac{i}{2} \frac{d k}{k}
$$

Therefore, for $\chi>\gamma(x)$

$$
\zeta(x)=A k^{-\frac{1}{2}} e^{ \pm i \int k\left(x^{\prime}\right) d x^{\prime}}
$$

In the classically allowed region $S_{0}= \pm \int_{x} \hbar k\left(x^{\prime}\right) d x^{\prime}$ counts the oscillations of the velocity wave function. An increase of $2 \pi \hbar$ corresponds to an additional phase of $2 \pi$. 
Similarly, in regions where $\chi<\gamma(x)$ we have:

$$
\zeta(x)=A \rho^{-\frac{1}{2}} e^{ \pm \int \rho\left(x^{\prime}\right) d x^{\prime}}
$$

For our first order expansion to be accurate we need that the magnitude of higher order terms decreases rapidly. We need $\left|\frac{d S_{0}}{d x}\right|>>\left|\hbar \frac{d S_{1}}{d x}\right|$ or $|k|>>\left|\frac{1}{2 k} \frac{d k}{d x}\right|$. The local deBroglie wavelength is $\lambda=2 \pi / k$. Therefore, $\left|\frac{\lambda}{4 \pi} \frac{d \lambda}{d x}\right|<<\lambda$, i.e. the change in $\lambda$ over a distance $\lambda / 4 \pi$ is small compared to $\lambda$. This holds when the velocity potential $\gamma(x)$ varies slowly and the momentum is nearly constant over several wavelengths.

Near the classical turning points the WKBJ solutions become invalid, because $k$ goes to zero here. We have to find a way to connect an oscillating solution to an exponential solution across a turning point if we want to solve barrier penetration problems or find bound states.

\subsection{Velocity potential $\gamma(\mathrm{x})$ and the bound states}

We want to find the velocity wave function in a given velocity potential well $\gamma(x)$. Assuming that the limit velocity of the particle is $\chi$ and that the classical turning points are $x_{1}$ and $x_{2}$, $x_{1}<x_{2}$, i.e. we have a velocity potential well with two sloping sides (Fig. 2).

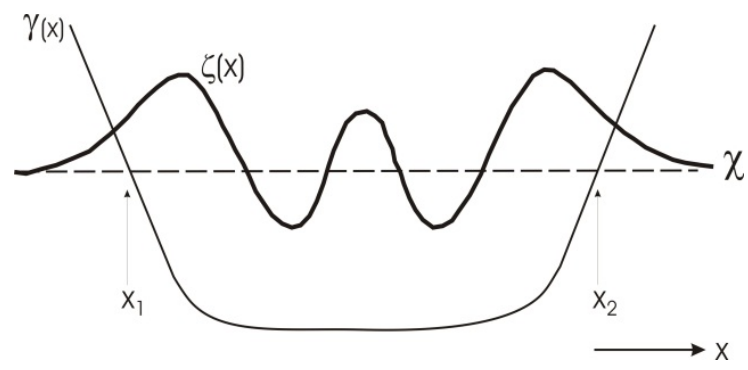

Figure 2. Bound state problem.

For $x<x_{1}$ the velocity wave function is of the form:

$$
\zeta_{1}(x)=A_{1} \rho^{-\frac{1}{2} e^{x} \rho\left(x^{\prime}\right) d x^{\prime}}
$$

For $x>x_{2}$ the velocity wave function is of the form:

$$
\zeta_{3}(x)=A_{3} \rho^{-\frac{1}{2}} e^{-\int \rho\left(x^{\prime}\right) d x^{\prime}}
$$


In the region between $x_{1}$ and $x_{2}$ it is of the form:

$$
\zeta_{2}(x)=A_{2} k^{-\frac{1}{2} e^{+i \int k\left(x^{\prime}\right) d x^{\prime}}}+A_{2}^{\prime} k^{-\frac{1}{2}} e^{-i \int k\left(x^{\prime}\right) d x^{\prime}}
$$

At $x=x_{1}$ and $x=x_{2}$ the velocity wave function $\zeta$ and its derivatives have to be continuous. Near $x_{1}$ and $x_{2}$ we expand the velocity potential well $\gamma(x)$ in a Taylor series expansion in $x$ and neglect all terms of order higher than 1 . Near $x_{1}$ we have $\gamma(x)=\chi-K_{1}\left(x-x_{1}\right)$, and near $x_{2}$ we have $\gamma(x)=\chi+K_{2}\left(x-x_{2}\right)$.

In the neighborhood of $x_{1}$ the time-independent Schrödinger equation then becomes:

$$
\frac{d^{2} \zeta}{d x^{2}}+\frac{2 \mu K_{1}}{\hbar^{2}}\left(x-x_{1}\right) \zeta=0
$$

and in the neighborhood of $x_{2}$ the time-independent Schrödinger equation becomes:

$$
\frac{d^{2} \zeta}{d x^{2}}-\frac{2 \mu K_{2}}{\hbar^{2}}\left(x-x_{2}\right) \zeta=0
$$

Let us define $z=-\left(\frac{2 \mu K_{1}}{\hbar^{2}}\right)^{\frac{1}{3}}\left(x-x_{1}\right)$. Then we obtain $\frac{d^{2} \zeta}{d z^{2}}-z \zeta=0$ near $x_{1}$. The solutions of this equation which vanish asymptotically as $z \rightarrow \infty$ or $x \rightarrow-\infty$ are the Airy functions. They are defined through:

$$
\operatorname{Ai}(z)=\frac{1}{\pi} \int_{0}^{\infty} \cos \left(\frac{s^{3}}{3}+s z\right) d s
$$

which for large $|z|$ has the asymptotic form

$$
A i(z) \sim \frac{1}{2 \sqrt{\pi} z^{\frac{1}{4}}} \exp \left(-\frac{2}{3} z^{\frac{3}{2}}\right), \quad(z>0)
$$

and

$$
A i(z) \sim \frac{1}{\sqrt{\pi}(-z)^{\frac{1}{4}}} \sin \left(\frac{2}{3}(-z)^{\frac{3}{2}}+\frac{\pi}{4}\right), \quad(z<0)
$$


If the limit velocity $\chi$ is high enough, the linear approximation to the velocity potential well remains valid over many wavelengths. The Airy functions can therefore be the connecting velocity wave functions through the turning point at $x_{1}$.

If we define $z=\left(\frac{2 \mu K_{2}}{\hbar^{2}}\right)^{\frac{1}{3}}\left(x-x_{2}\right)$ then we find $\frac{d^{2} \zeta}{d z^{2}}-z \zeta=0$ near $x=x_{2}$ and the Airy functions can also be the connecting velocity wave functions through the turning point at $x_{2}$. Here $z \rightarrow \infty$ or $x \rightarrow \infty$.

In the neighborhood of $x_{1}$ we have

$$
k^{2}=-\rho^{2}=\left(\frac{2 \mu K_{1}}{\hbar^{2}}\right)^{\frac{1}{3}}\left(x-x_{1}\right)=-\left(\frac{2 \mu K_{1}}{\hbar^{2}}\right)^{\frac{1}{3}} z
$$

Therefore

$$
\int_{x_{1}}^{x} \rho d x^{\prime}=\left(\frac{2 \mu K_{1}}{\hbar^{2}}\right)^{\frac{1}{3}} \int_{x_{1}}^{x} \sqrt{z} d x^{\prime}=-\int_{0}^{x} \sqrt{z^{\prime}} d z^{\prime}=-\frac{2}{3} z^{\frac{3}{2}}
$$

Similarly

$$
\int_{x_{1}}^{x} k d x^{\prime}=\left(\frac{2 \mu K_{1}}{\hbar^{2}}\right)^{\frac{1}{3}} \int_{x_{1}}^{x} \sqrt{-z} d x^{\prime}=-\int_{0}^{x} \sqrt{-z^{\prime}} d z^{\prime}=\frac{2}{3}(-z)^{\frac{3}{2}}
$$

By comparing this with the asymptotic forms of the Airy functions we note that

$$
\zeta_{1}(x)=A_{1} \rho^{-\frac{1}{2}} e^{+\int_{x_{1}}^{x} \rho\left(x^{\prime}\right) d x^{\prime}}\left(x<x_{1}\right)
$$

must continue on the right side as

$$
\zeta_{2}(x)=2 A_{1} k^{-\frac{1}{2}} \sin \left(\int_{x_{1}}^{x} k d x^{\prime}+\frac{\pi}{4}\right)\left(x>x_{1}\right)
$$

In the neighborhood of $x_{2}$ we similarly find that 


$$
\zeta_{3}(x)=A_{3} \rho^{-\frac{1}{2}} e^{-\int_{x_{2}}^{x} \rho\left(x^{\prime}\right) d x^{\prime}}\left(x>x_{2}\right)
$$

must continue in region 2 as

$$
\zeta_{2}(x)=2 A_{3} k^{-\frac{1}{2}} \sin \left(\int_{x}^{x_{2}} k d x^{\prime}+\frac{\pi}{4}\right)\left(x<x_{2}\right)
$$

Both expressions for $\zeta_{2}(x)$ are approximations to the same eigenfunction. We therefore need

$$
2 A_{1} k^{-\frac{1}{2}} \sin \left(\int_{x_{1}}^{x} k d x^{\prime}+\frac{\pi}{4}\right)=2 A_{3} k^{-\frac{1}{2}} \sin \left(\int_{x}^{x_{2}} k d x^{\prime}+\frac{\pi}{4}\right)
$$

For (79) to be satisfied, the amplitudes of each side must have the same magnitude, and the phases must be the same modulo $\pi$ :

$$
\begin{aligned}
& \left|A_{1}\right|=\left|A_{3}\right| \\
& \int_{x_{1}}^{x} k d x^{\prime}+\frac{\pi}{4}=-\int_{x}^{x_{2}} k d x^{\prime}-\frac{\pi}{4}+n \pi
\end{aligned}
$$

Knowing that $\int_{x_{1}}^{x_{2}}=\int_{x_{1}}^{x}+\int_{x}^{x_{2}}$, we have

$$
\int_{x_{1}}^{x_{2}} k d x^{\prime}=\left(n-\frac{1}{2}\right) \pi, \quad n=1,2,3, \ldots
$$

This can be re-written as

$$
\int_{x_{1}}^{x_{2}} \Pi d x=\left(n-\frac{1}{2}\right) \frac{h}{2} \text { or } \oint \Pi d x=\left(n-\frac{1}{2}\right) h
$$

with 


$$
\Pi=[2 \mu(\chi-\gamma(x))]^{1 / 2}=[2 \mu U(x)]^{1 / 2}=m c\left[2 \frac{U(x)}{c}\right]^{1 / 2}
$$

Here $\oint$ denote an integral over one complete cycle of the classical motion. The WKBJ method for $\gamma(x)$ velocity potential well with soft walls, therefore, leads to a Wilson-Sommerfeld type quantization rule except that $n$ is replaced by $n-1 / 2$. It leads to a quantization of the complex velocity $U(x)$.

The factor of $\pi / 2$ arises here due to the two phase changes of $\pi / 4$ at $x_{1}$ and $x_{2}$. In case where only one of the walls is soft and the other is infinitely steep the factor of $1 / 2$ is replaced by $1 / 4$ in (81). If both walls are infinitely steep, the factor of $1 / 2$ in (81) is replaced by 0 .

WKBJ approximation is a semi classical approximation, since it is expected to be most useful in the nearly classical limit of large quantum numbers. The method will not be good for, say, lowest limit velocity states $\chi$, so in order to overcome this shortcomings there is a need for a modified semi classical quantization condition. For oscillations between the two classical turning points $x_{1}$ and $x_{2}$, we obtain the semi classical quantization condition by requiring that the total phase during one period of oscillation to be an integral multiple of $2 \pi$; [15] such that

$$
2 \int_{x_{1}}^{x_{2}} k d x^{\prime}+\phi_{1}+\phi_{2}=2 \pi n
$$

where $\phi_{1}$ is the phase loss due to reflection at the classical turning point $x_{1}$ and $\phi_{2}$ is the phase loss due to reflection at $x_{2}$. Taking $\phi_{1}$ and $\phi_{2}$ to be equal to $\pi / 2$ leads to the modified semiclassical quantization rule, i.e.

$$
\int_{x_{1}}^{x_{2}} k d x^{\prime}=\left(n-\frac{m}{4}\right) \pi
$$

where mis the Maslov index [15], which denotes the total phase loss during one period in units of $\pi / 2$. It contains contributions from the phase losses $\phi_{1}$ and $\phi_{2}$ due to reflections at points $x_{1}$ and $x_{2}$, respectively. It is pertinent to note that taking $\phi_{1}=\phi_{2}=\pi / 2$ and an integer Maslov index $m=2$ in (85), we have the familiar semi classical quantization rule, i.e. (81).

Let us apply the constraint equation (81) to an harmonic oscillator. The condition then is (passing without loss of generality to the limits $-a$ to $+a$ ) 


$$
\int_{-a}^{+a}[2 \mu(\chi-\omega x)]^{1 / 2} d x=\left(n-\frac{1}{2}\right) \pi \hbar
$$

where the energy $W_{c}$ of the oscillator $U(x)$ with the pulsation $\omega$ writes

$$
W_{c}=\frac{1}{2} m \omega^{2} x^{2}=\frac{1}{2} m \gamma^{2}(x)
$$

and we get the expression for the $x$ dependence of the velocity term, $\gamma(x)=\omega x$.

Theleft side term of (86) is an elementary integral and we find:

$$
(\chi+v)^{3 / 2}-(\chi-v)^{3 / 2}=\frac{3 \pi}{2 a(2 \mu)^{1 / 2}}\left(n-\frac{1}{2}\right) \hbar v
$$

where $v=\omega a$ is the liniar velocity (see the graphic in Fig. 3).

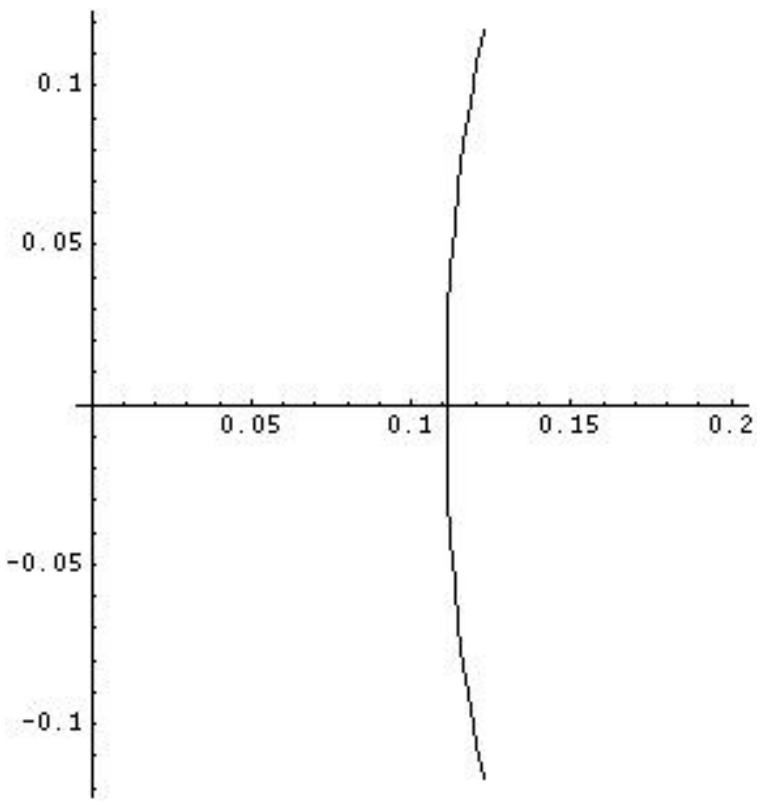

Figure 3. Dependence of the limit velocity $x$ on the linear velocity $u$. 
We try to estimate a value for the limit velocity $\chi$. Let us expand the left side term of (88) in series and keep the first term. If we replace $\mu$ from (56) and take $a=\Lambda$ (the Compton length), we get:

$$
\chi \approx \frac{\pi^{2} \hbar^{2}}{8 \mu a^{2}}\left(n-\frac{1}{2}\right)^{2}=\frac{\pi^{2}}{32}(2 n-1)^{2} c=\left\{\frac{\pi^{2}}{32} c, 9 \frac{\pi^{2}}{32} c, 25 \frac{\pi^{2}}{32} c, \ldots\right\}
$$

It is interesting to note that only the first velocity in (89) is less than the velocity of light, $c$.

Let us analyze now, one more bound state, the velocity wave function in a given velocity double well potential $\gamma(x)$.

We begin by deriving a quantization condition for region 2 analogous to (81). Again, applying the boundary condition for region 1 leaves only the exponentially growing solution. Applying the connection formula at $x_{1}$ then gives an expression for the velocity wave function in region 2:

$$
\zeta_{1}(x)=2 A k^{-1 / 2} \sin \left[\int_{x_{1}}^{x} k d x^{\prime}+\frac{\pi}{4}\right]
$$

However, the solution in region 3 must have both growing and decaying solutions present. Considering the region 3 solutions in terms of $x_{2}$ and letting $B_{L}$ and $C_{L}$ be the amplitudes of the decaying and growing solutions respectively, the connection formulas give another expression for the velocity wave function in region 2 :

$$
\zeta_{2}(x)=2 B_{L} k^{-1 / 2} \cos \theta+C_{L} k^{-1 / 2} \sin \theta
$$

with

$$
\theta=\int_{x}^{x_{2}} k d x^{\prime}-\frac{\pi}{4}
$$

We equate the two expressions (90), (91) for the velocity function in region 2 and cancel common factors giving

$$
2 A \sin \left[\int_{x_{1}}^{x} k d x^{\prime}+\frac{\pi}{4}\right]=2 B_{L} \cos \theta+C_{L} \sin \theta
$$


Using trigonometric identities to simplify the right hand side, gives

$$
2 A \sin \left[\int_{x_{1}}^{x} k d x^{\prime}+\frac{\pi}{4}\right]=\left(4 B_{L}^{2}+C_{L}^{2}\right)^{1 / 2} \sin \left(\theta+\frac{\pi}{2}-\phi_{L}\right)
$$

where

$$
\phi_{L}=\cos ^{-1}\left[\frac{2 B_{L}}{\left(4 B_{L}^{2}+C_{L}^{2}\right)^{1 / 2}}\right]
$$

The magnitude of the sin function must be equal, and the magnitude of the phases must be equal modulo $\pi$ :

$$
\begin{gathered}
4 A^{2}=4 B_{L}^{2}+C_{L}^{2} \\
\int_{x_{1}}^{x} k d x^{\prime}+\frac{\pi}{4}=-\int_{x}^{x_{2}} k d x^{\prime}-\frac{\pi}{4}+\phi_{L}+n \pi
\end{gathered}
$$

Simplifying and combining the integrals gives the quantization condition for region 2:

$$
\theta_{12} \equiv \int_{x_{1}}^{x_{2}} k d x=\pi\left(n-\frac{1}{2}\right)+\phi_{L}
$$

with $n=1,2, \ldots$.

A similar treatment for the turning point $x_{3}$ yields the condition for region 4 :

$$
\theta_{34} \equiv \int_{x_{3}}^{x_{4}} k d x=\pi\left(m-\frac{1}{2}\right)+\phi_{R}
$$

with $m=1,2, \ldots$ and $\phi_{R}$ given by:

$$
\phi_{R}=\cos ^{-1}\left[\frac{2 C_{R}}{\left(4 C_{R}^{2}+B_{R}^{2}\right)^{1 / 2}}\right]
$$


where $B_{R}$ and $C_{R}$ are the amplitudes of the decaying and growing region 3 solutions in terms of $x_{3}$.

We now have the quantization conditions $(98,99)$ for regions 2 and 4 , but they contain the free parameters $\phi_{L}$ and $\phi_{R}$. To eliminate these free parameters, we consider the WKBJ solution in region 3. The coefficients $B_{L}, C_{L}, B_{R}, C_{R}$ define two expressions for solution, which must be equal:

$$
\begin{aligned}
& \zeta_{3}=B_{L} \rho^{-1 / 2} \exp \left[-\int_{x_{2}}^{x} \rho d x^{\prime}\right]+C_{L} \rho^{-1 / 2} \exp \left[\int_{x_{2}}^{x} \rho d x^{\prime}\right] \\
& \zeta_{3}=B_{R} \rho^{-1 / 2} \exp \left[\int_{x}^{x_{3}} \rho d x^{\prime}\right]+C_{R} \rho^{-1 / 2} \exp \left[-\int_{x}^{x_{3}} \rho d x^{\prime}\right]
\end{aligned}
$$

Equations (101) and (102) each contain a term that grows exponentially with $x$ and a term that decays exponentially with $x$. Equating the growing terms from each equation and the decaying term from each equation gives two constraints:

$$
\begin{aligned}
& B_{L} \exp \left[-\int_{x_{2}}^{x} \rho d x^{\prime}\right]=B_{R} \exp \left[\int_{x}^{x_{3}} \rho d x^{\prime}\right] \\
& C_{L} \exp \left[\int_{x_{2}}^{x} \rho d x^{\prime}\right]=C_{R} \exp \left[-\int_{x}^{x_{3}} \rho d x^{\prime}\right]
\end{aligned}
$$

Combining the integrals in these constraints gives

$$
\frac{B_{L}}{B_{R}}=\frac{C_{R}}{C_{L}}=\exp \left(\theta_{23}\right)
$$

with

$$
\theta_{23} \equiv \int_{x_{2}}^{x_{3}} \rho d x^{\prime}
$$


The constraints $(98,99,105)$ may be combined to give a single quantization condition for the allowed WKBJ velocity limits $\chi$ for a double-well velocity potential $\gamma(x)$. Applying trigonometric identities to (95) and (100), and plugging into (105) gives

$$
\tan \phi_{L} \tan \phi_{R}=\left(\frac{C_{L}}{2 B_{L}}\right)\left(\frac{B_{R}}{2 C_{R}}\right)=\frac{1}{4} \exp \left(-2 \theta_{23}\right)
$$

Equation (107) may be combined with (98) and (99) to give the WKBJ quantization condition for a double-well potential in terms of the phase integrals $\theta_{12}$ and $\theta_{34}$ :

$$
\operatorname{ctg} \theta_{12} \operatorname{ctg} \theta_{34}=\frac{1}{4} \exp \left(-2 \theta_{23}\right)
$$

confirming the results given in [16].

Equation (108) is a nonlinear constraint approximately determining the allowed velocity levels $\chi$ of a double-well velocity potential $\gamma(x)$ (see Fig. 4) and can be written (taking $\phi_{R}=\phi_{L}=\pi / 4$ in (98) and (99), i.e. the velocity quarter-wave shift in the connection formulas, which is known to optimize the tunneling effect between two oscillating waves [17] ) as :

$$
\frac{1}{\hbar} \int_{x_{2}}^{x_{3}}[2 \mu(\gamma(x)-\chi)]^{1 / 2} d x=\ln \left\{4 \cdot \operatorname{ctg}\left[\pi\left(n-\frac{1}{2}\right)+\phi_{L}\right] \cdot \operatorname{ctg}\left[\pi\left(m-\frac{1}{2}\right)+\phi_{R}\right]\right\}^{-1 / 2}=-\ln 2 \quad \mathrm{~m}, \mathrm{n}=1,2,3, \ldots
$$

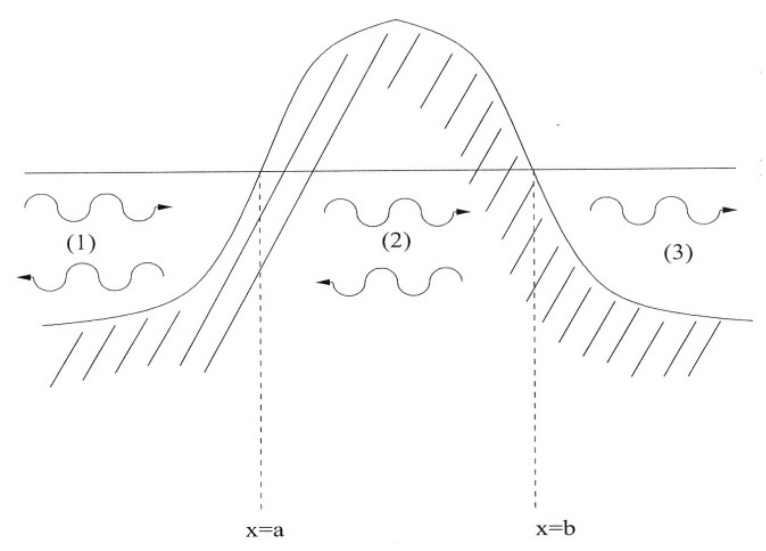

Figure 4. Tunneling potential barrier.

In terms of the momentum $\Pi$ we have : 


$$
\int_{x_{2}}^{x_{3}} \Pi d x=-\hbar \ln 2 \text { or } \oint \Pi d x=-2 \hbar \ln 2
$$

where $\oint$ denotes an integral over one complete cycle of the classical motion, this time

$$
\Pi=[2 \mu(\gamma(x)-\chi)]^{1 / 2}=[2 \mu U(x)]^{1 / 2}=m c\left[2 \frac{U(x)}{c}\right]^{1 / 2}
$$

since $\gamma(x)>\chi$ for the integration limits, i.e. region 3 (see Fig. 5). We get again a quantization of the complex velocity $U(x)$, where the levels are equally spaced at a value of $\hbar \ln 2$.

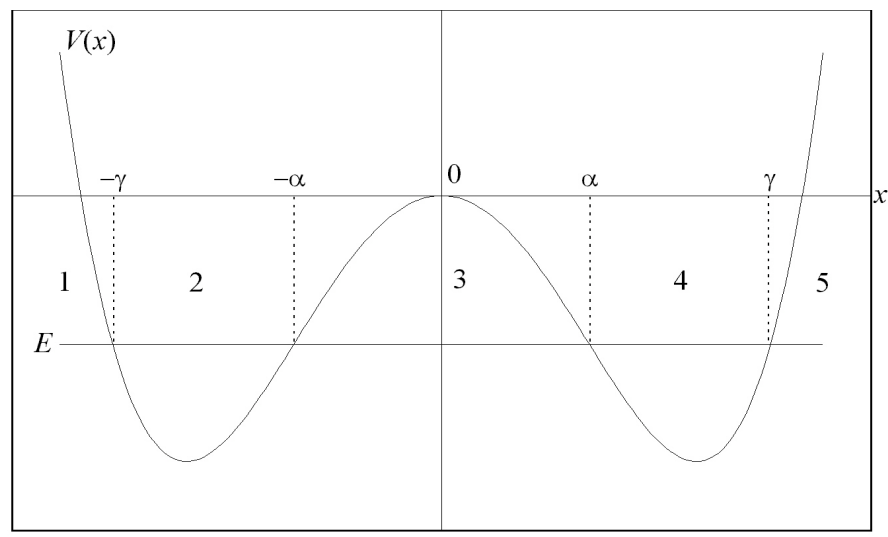

Figure 5. Schematic diagram of a double-well potential with three forbidden regions $(1,3,5)$ and two allowed regions $(2,4)$

In 1961, Landauer [18] discussed the limitation of the efficiency of a computer imposed by physical laws. In particular he argued that, according to the second law of thermodynamics, the erasure of one bit of information requires a minimal heat generation $k_{B} T \ln 2$, where $k_{B}$ is Boltzmann's constant and $T$ is the temperature at which one erases. Its argument runs as follows. Since erasure is a logical function that does not have a single-valued inverse it must be associated with physical irreversibility and therefore requires heat dissipation. A bit has one degree of freedom and so the heat dissipation should be of order $k_{B} T$. Now, since before erasure a bit can be in any of the two possible states and after erasure it can only be in one state, this implies a change in information entropy of an amount $-k_{B} \ln 2$.

The one-to-one dynamics of Hamiltonian systems [19] implies that when a bit is erased the information which it contains has to go somewhere. If the information goes into observable 
degrees of freedom of the computer, such as another bit, then it has not been erased but merely moved; but if it goes into unobservable degrees of freedom such as the microscopic motion of molecules it results in an increase of entropy of at least $k_{B} \ln 2$.

Inspired by such studies, a considerable amount of work has been made on the thermodynamics of information processing, which include Maxwell's demon problem [20], reversible computation [21], the proposal of the algorithmic entropy [22] and so on.

Here, considering a double-well velocity potential $\gamma(x)$ and the velocity quarter-wave shift in the connection formulas, a quanta of $\hbar \ln 2$ for the complex velocity $U(x)$ of the moving Newtonian 'fluid' occurs. It can be argued that it can be put into a one-to-one correspondence to the quanta of information Landauer and other authors discussed about [23, 24].

Furthermore, one gets an interesting result when taking $\phi_{R}=\phi_{L}=\pi / 2$, i.e. the velocity halfwave shift in the connection formulas, when singularities occur in (II.66). We try to solve this case by making use of the vortices theory. Benard in 1908 was the first to investigate the appearance of vortices behind a body moving in a fluid [12]. The body he used was a cylinder. He observed that at a high enough fluid velocity (or Reynolds number based on the cylinder diameter), which depends on the viscosity and width of the body, vortices start to shed behind the cylinder, alternatively from the top and the bottom of the cylinder.

Consequently, we write (109) in the form

$$
\int_{x}[2 \mu(\gamma(x)-\chi)]^{1 / 2} d x=-\hbar \ln 2-\hbar \ln \left\{\operatorname{tg}\left[\frac{\pi}{l}\left(x-x_{0}\right)\right]\right\}
$$

where we use $\operatorname{ctg}(\alpha+\pi / 2)=-\operatorname{tg}(\alpha)$, take $m=n$, make the notations $x=n l, \quad x_{0}=l / 2$ and consider again the one-dimensional case, motion along the Ox axis.

Solving (112) one gets

$$
U(x)=c\left(1+\operatorname{ctg}^{2}\left[\frac{2 \pi}{\Lambda}\left(x-x_{0}\right)\right]\right)
$$

where we assume $l=\Lambda$ (the Compton length), $U(x)=[\gamma(x)-\chi] / 2 \pi^{2}$ and replace $\mu=m^{2} c$, where $c$ is the velocity of light. When plotting (113) (see Fig. 6) we see that indeed, singularities are obtained for $x-x_{0}=\Lambda / 2$ and for $x-x_{0}=\Lambda / 4$ we get for $U(x)$ minima of value the velocity of light, $c$.

Usually, at some distance behind a body placed in a fluid, vortices are arranged at a definite distance $l$ apart and with a definite separation $h$ between the two rows. The senses of the rotation in the two rows are opposite (see Fig. 7). 


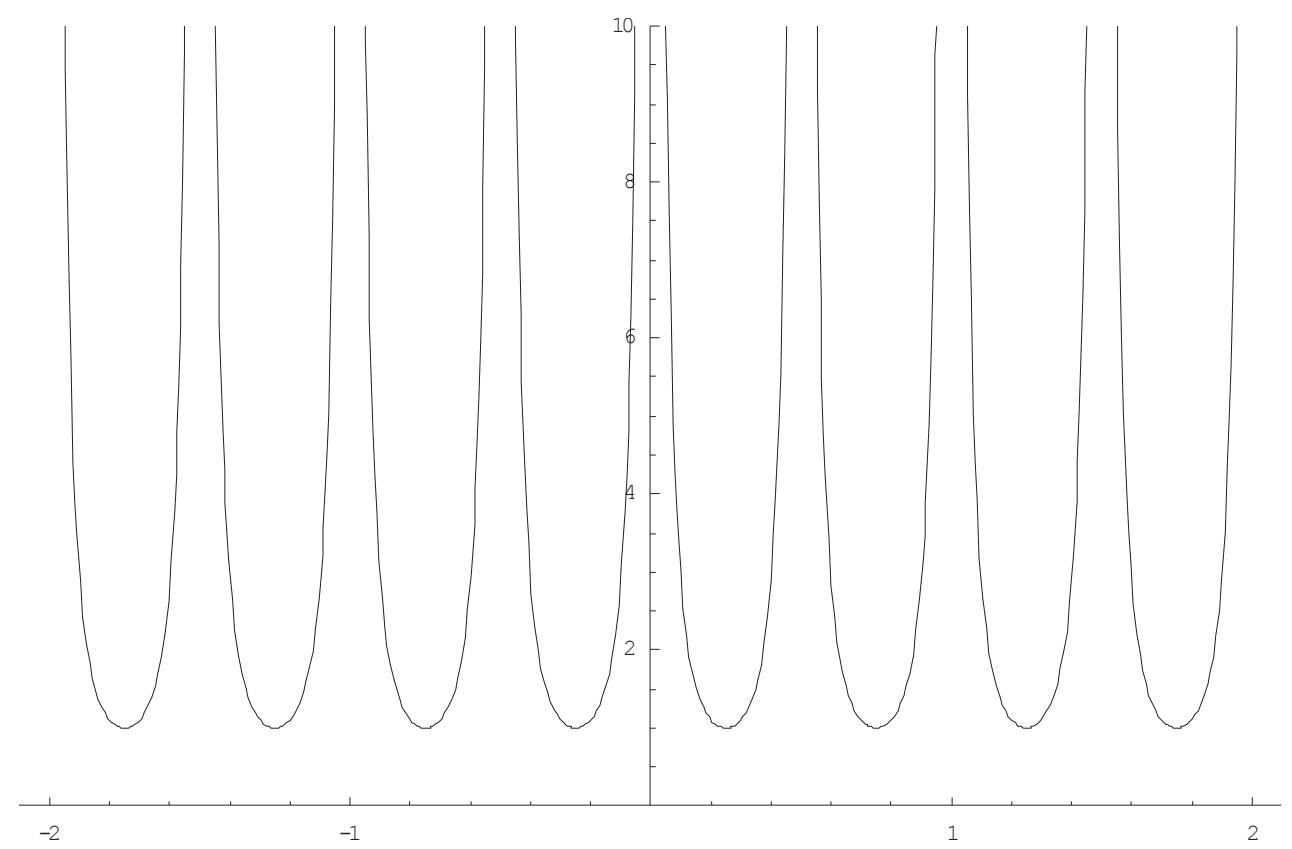

Figure 6. The complex velocity $U(x)$ singularities' distribution along the Ox axis.

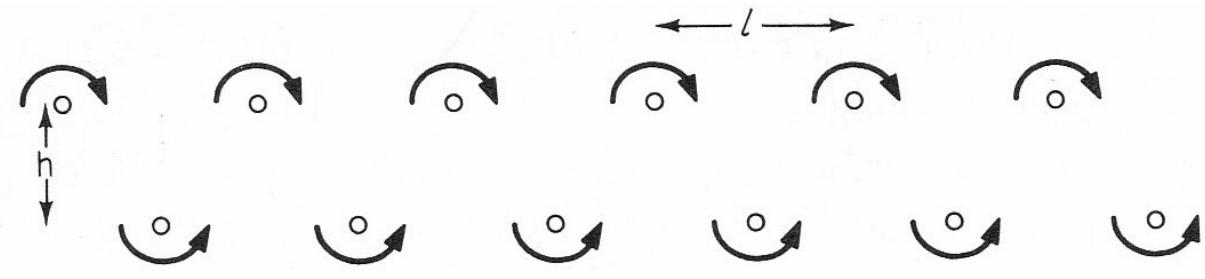

Figure 7. Von Karman vortex streets.

In 1912 von Karman expounded a theory of such vortex streets and the drag which a cylinder would experience due to their formation [12]. Since we considered here the one-dimensional case, we get the solution of a single row of rectilinear vortices, which has already been referred to as characterizing a surface of discontinuity (see Fig. 8).

A typical bound state in a double-well velocity potential has two classically allowed regions, where the velocity potential $\gamma(x)$ is less than the limit velocity $\chi$. These regions are separated by a classically forbidden region, or barrier, where the velocity potential is larger than the limit velocity. As we can see, quantum mechanics predicts that a velocity wave $\zeta(x)$ travelling in such a potential is most likely to be found in the allowed regions. However, unlike classical mechanics, quantum mechanics predicts that this velocity wave can also be found in the 


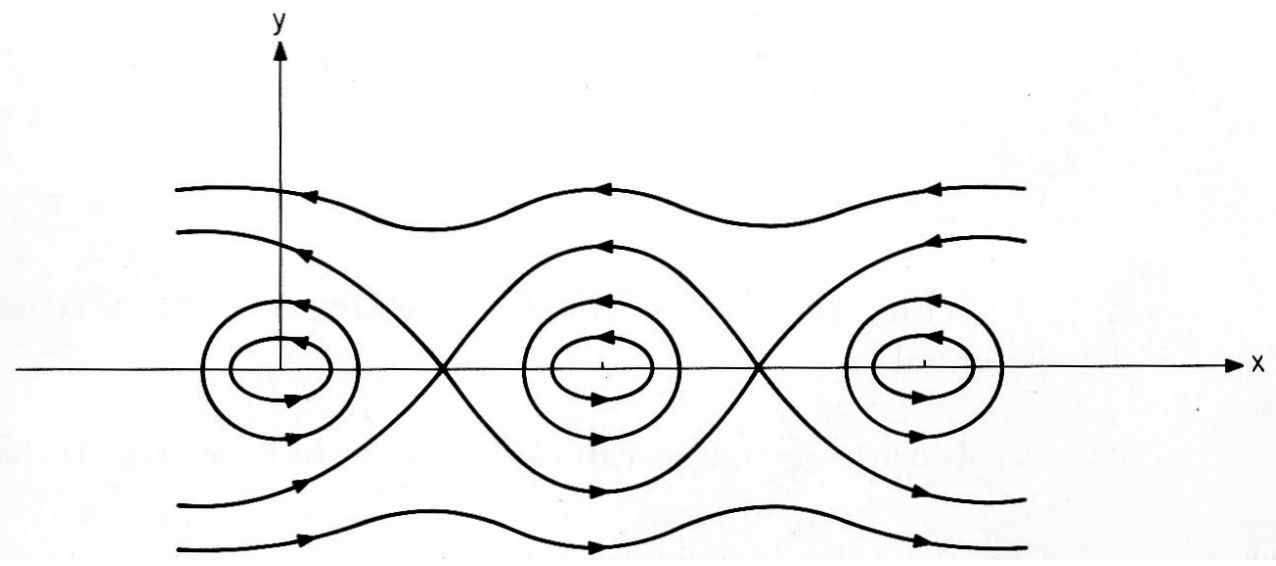

Figure 8. Single row of rectilinear vortices.

forbidden region. This uniquely quantum mechanical behavior allows a velocity wave, initially localized in one potential well, to penetrate through the barrier, into the other well (as we will see in what follows).

\subsection{Velocity potential $\gamma(x)$ and the quantum barrier}

We already know at the points where $\chi-\gamma(x)=0$, special treatment is required because $k$ is singular. The way of handling the solution near the turning point is a little bit more technical, but the basic idea is that we have a solution to the left and to the right of the turning point, and one needs a formula that interpolates between them. In other words, in the vicinity of the turning point one approximates $\sqrt{2 \mu(\chi-\gamma(x)) / \hbar^{2}}$ by a straight line over a small interval and solves TISE (time independent Schrodinger equation) exactly. This leads to the following connection formulas:

Barrier to the right ( $\mathrm{x}=\mathrm{b}$ turning point $)$

$$
\begin{gathered}
\frac{2}{\sqrt{k}} \cos \left[\int_{x}^{b} k(x) d x-\frac{\pi}{4}\right] \Leftrightarrow \frac{1}{\sqrt{\rho}} e^{-\int_{b}^{x} \rho(x) d x} \\
\frac{1}{\sqrt{k}} \sin \left[\int_{x}^{b} k(x) d x-\frac{\pi}{4}\right] \Leftrightarrow-\frac{1}{\sqrt{\rho}} e^{\int^{b} \rho(x) d x}
\end{gathered}
$$

Barrier to the left ( $x=$ a turning point $)$ 


$$
\begin{aligned}
& \frac{2}{\sqrt{k}} \cos \left[\int_{a}^{x} k(x) d x-\frac{\pi}{4}\right] \Leftrightarrow \frac{1}{\sqrt{\rho}} e^{-\int^{a} \rho(x) d x} \\
& \frac{1}{\sqrt{k}} \sin \left[\int_{a}^{x} k(x) d x-\frac{\pi}{4}\right] \Leftrightarrow-\frac{1}{\sqrt{\rho}} e^{\int^{x} \rho(x) d x}
\end{aligned}
$$

The connection formulas enable us to obtain relationships between the solutions in a region at some distance to the right of the turning point with those in a region at some distance to the left [25-27].

One of the most important problems to which connection formulas apply is that of the penetration of a potential barrier. The barrier is shown in Fig. 4 and the limit velocity $\chi$ is such that the turning points are at $x=a$ and $x=b$.

Suppose that the motion is incident from the left. Some waves will be reflected and some transmitted, so that in region III we will have:

$$
\zeta_{3}(x)=\frac{1}{\sqrt{k}} e^{i \int_{b}^{x} k d x-i \frac{\pi}{4}}=\frac{1}{\sqrt{k}} \cos \left[\int_{b}^{x} k d x-\frac{\pi}{4}\right]+\frac{i}{\sqrt{k}} \sin \left[\int_{b}^{x} k d x-\frac{\pi}{4}\right]
$$

The phase factor is included for convenience of applying the connection formulas.

In region II (using (114) and (115) on (118)) we have:

$$
\zeta_{2}(x)=\frac{1}{2} \frac{1}{\sqrt{\rho}} e^{-\int^{b} \rho d x}-i \frac{1}{\sqrt{\rho}} e^{\int^{b} \rho d x}
$$

Now using

$$
\int_{x}^{b} \rho d x=\int_{x}^{a} \rho d x+\int_{a}^{b} \rho d x=-\int_{a}^{x} \rho d x+\alpha
$$

we can write

$$
\zeta_{2}(x)=\frac{1}{2} \frac{1}{\sqrt{\rho}} e^{\int^{a} \rho d x} e^{-\alpha}-i \frac{1}{\sqrt{\rho}} e^{-\int_{a}^{x} \rho d x} e^{\alpha}
$$


Again, using the connection formulas for the case barrier to the right (using (116) and (117) on (121)), we get for region I:

$$
\begin{aligned}
\zeta_{1}(x)= & \frac{1}{2} e^{-\alpha} \frac{1}{\sqrt{k}} \sin \left[\int_{x}^{a} k d x-\frac{\pi}{4}\right]-i \frac{2}{\sqrt{k}} e^{\alpha} \cos \left[\int_{x}^{a} k d x-\frac{\pi}{4}\right]= \\
= & \frac{1}{2} \frac{1}{\sqrt{k}} e^{-\alpha} \sin (u)-i \frac{2}{\sqrt{k}} e^{\alpha} \cos (u)= \\
= & -\frac{i}{\sqrt{k}}\left[e^{i u}\left(e^{\alpha}+\frac{1}{4} e^{-\alpha}\right)+e^{-i u}\left(e^{\alpha}-\frac{1}{4} e^{-\alpha}\right)\right]
\end{aligned}
$$

Hence

$$
\left\{\begin{array}{l}
\zeta_{1}^{i n c}(x)=-\frac{i}{\sqrt{k}}\left(e^{\alpha}+\frac{1}{4} e^{-\alpha}\right) e^{i \int k x d x-i \frac{\pi}{4}} \\
\zeta_{1}^{r e f}(x)=-\frac{i}{\sqrt{k}}\left(e^{\alpha}-\frac{1}{4} e^{-\alpha}\right) e^{-i \int^{a} k d x+i \frac{\pi}{4}}
\end{array}\right.
$$

Having obtained the expression for $\zeta_{1}^{\text {inc }}(x)$ and $\zeta_{1}^{\text {ref }}(x)$ we are now in position to calculate the transmission coefficient using:

$$
T=\left|\frac{\zeta_{3}(x)}{\zeta_{1}^{\text {inc }}(x)}\right|^{2}=\frac{e^{-2 \alpha}}{\left(1+\frac{1}{4} e^{-2 \alpha}\right)^{2}}
$$

To summarize, for a barrier with large attenuation $e^{-2 \alpha} \rightarrow 0$, the tunneling probability equals

$$
T=\frac{e^{-2 \alpha}}{\left(1+\frac{1}{4} e^{-2 \alpha}\right)^{2}} \approx e^{-2 \alpha}=\exp \left(-\frac{2}{\hbar} \int_{a}^{b} \rho d x\right)=\exp \left(-\frac{2}{\hbar} \int_{a}^{b}[2 \mu(\gamma(x)-\chi)]^{1 / 2} d x\right)
$$

The reflection coefficient is:

$$
R=\left|\frac{\zeta_{1}^{r e f}(x)}{\zeta_{1}^{\text {inc }}(x)}\right|^{2} \cong \frac{\left(e^{\alpha}-\frac{1}{4} e^{-\alpha}\right)^{2}}{\left(e^{\alpha}+\frac{1}{4} e^{-\alpha}\right)^{2}}, \quad T+R=1
$$


and also in the same large attenuation limit, we have:

$$
R \approx 1-e^{-2 \alpha}=1-\exp \left(-\frac{2}{\hbar} \int_{a}^{b} \rho d x\right)=1-\exp \left(-\frac{2}{\hbar} \int_{a}^{b}[2 \mu(\gamma(x)-\chi)]^{1 / 2} d x\right)
$$

One can see from (125) and (127) thatthe velocity wave $\zeta(x)$ on small distances, with the same order of magnitude as $\Lambda$, may be influenced by $U(x)$,i.e. it can be transmitted, attenuated or reflected at this scale length. In other words, we get from the calculus, that the velocity field $V$ is indeed transported by the motion of the 'Newtonian fluid' particles with the velocity $U(x)$ (the imaginary part of the complex velocity [13]).

\section{Casimir type effect in scale relativity theory}

In recent years, new and exciting advances in experimental techniques [28] prompted a great revival of interest in the Casimir effect, over fifty years after its theoretical discovery (for a recent review on both theoretical and experimental aspects of the Casimir effect, see Refs. [29-31]). As is well known, this phenomenon is a manifestation of the zero-point fluctuations of the electromagnetic field: it is a purely quantum effect and it constitutes one of the rare instances of quantum phenomena on a macroscopic scale.

In his famous paper, Casimir evaluated the force between two parallel, electrically neutral, perfectly reflecting plane mirrors, placed a distance $L$ apart, and found it to be attractive and of a magnitude equal to:

$$
F_{C}=\frac{\hbar c \pi^{2} A}{240 L^{4}}
$$

Here, $A$ is the area of the mirrors, which is supposed to be much larger than $L^{2}$, so that edge effects become negligible. The associated energy $E_{C}$

$$
E_{C}=-\frac{\hbar c \pi^{2} A}{720 L^{3}}
$$

can be interpreted as representing the shift in the zero-point energy of the electromagnetic field, between the mirrors, when they are adiabatically moved towards each other starting from an infinite distance. The Casimir force is indeed the dominant interaction between neutral bodies at the micrometer or submicrometer scales, and by modern experimental techniques it has now been measured with an accuracy of a few percent (see [28] and references therein). 
Since this effect arises from long-range correlations between the dipole moments of the atoms forming the walls of the cavity, that are induced by coupling with the fluctuating electromagnetic field, the Casimir energy depends in general on the geometric features of the cavity. For example, we see from (129) that, in the simple case of two parallel slabs, the Casimir energy $E_{C}$ is negative and is not proportional to the volume of the cavity, as would be the case for an extensive quantity, but actually depends separately on the area and distance of the slabs. Indeed, the dependence of $E_{C}$ on the geometry of the cavity can reach the point where it turns from negative to positive, leading to repulsive forces on the walls. For example [29], in the case of a cavity with the shape of a parallelepiped, the sign of $E_{C}$ depends on the ratios among the sides, while in the case of a sphere it has long been thought to be positive. It is difficult to give a simple intuitive explanation of these shape effects, as they hinge on a delicate process of renormalization, in which the finite final value of the Casimir energy is typically expressed as a difference among infinite positive quantities. In fact, there exists a debate, in the current literature, whether some of these results are true or false, being artifacts resulting from an oversimplification in the treatment of the walls [33].

There are three well-known technical types of derivation of the Casimir force for different geometries including the simplest geometry of two parallel, uncharged, perfectly conducting plates firstly explored by Casimir. One modern method is the quantum field theoretical approach based on the appropriate Green's function of the geometry of problem [34]. The other technical type is the dimensional regularization method that involves the mathematical complications of the Riemann zeta function and the analytical continuation [34]. The last (the most elementary/the simplest) method is based on modes summation by using the EulerMaclurian integral formula [35-37].

The problem of finding the Casimir force, not only for the simplest geometry of two plates or rectangular prism, that we want to study here, but also for other more complicated geometries, indispensably/automatically involves some infinities/irregularities; thus, one should regularize the calculation for arriving at the desired finite physical result(s). In the Green' function method, one uses the subtraction of two terms (two Green's functions) to do the required regularization. In the dimensional regularization method, although there isn't an explicit subtraction for the regularization of the problem, as is clear from its name, the calculation is regularized dimensionally by going to a complex plane with a mathematically complicated/ ambiguous approach. In the simplest method in which the Euler-Maclurian formula is used, the regularization is performed by the subtraction of the zero-point energy of the free space (no plates) from the energy expression under consideration/calculation (e.g. summation of the interior and exterior zero-point energies of the two parallel plates).

Navier-Stokes equations in scale relativity theory predict that the (vector) velocity field $V$ and/ or the (scalar) density field $\rho$, on small distances (the same magnitude as the Compton length) behave like a wave function and are transported by the motion of the Newtonian fluid with velocity $U$.

Furthermore, when considering vacuum from the Casimir cavity, a non-differentiable, Newtonian, 2D non-coherent quantum fluid whose entities (cvasi-particles) assimilated to vortex-type objects, initially non-coherent, become coherent (the coherence of the quantum 
fluid reduces to its ordering in vortex streets) due to the constraints induced by the presence of slabs. Casimir type forces are derived which are in good agreement with other theoretical results and experimental data, for both cases: two metallic slabs, parallel to each other, placed at a distance $d$ apart, that constitute the plates of the cavity and a rectangle of sides $d_{1}, d$.

In other words, non-differentiability and coherence of the quantum fluid due to constraints generate pressure along the $O x$ and $O y$ axis.

For viscous compressible fluids, Navier-Stokes equations

$$
\rho \frac{D v}{D t}=\rho \boldsymbol{X}-\nabla p+\mu \nabla^{2} \boldsymbol{v}+\frac{\mu}{3} \nabla(\nabla \cdot v)
$$

together with the equation of continuity

$$
\frac{D \rho}{D t}+\rho \nabla \cdot \boldsymbol{v}=0
$$

where $\rho$ is the density, $v$ the velocity of the fluid, $X$ the body force, $p$ the pressure, $\mu$ the shear viscosity and $D / D t \equiv d / d t+v \cdot \nabla$ the Eulerian derivative, apply to Newtonian (or near) fluids, that is, to fluids in which the stress is linearly related to the rate of strain (as will be assumed further in this section) [12].

Let us see first, what happens with the set of equations (130) and (131), if one considers that the space-time, where particles move, changes from classical to non-differentiable.

We already know, according to Nottale [11], that a transition from classical (differentiable) mechanics to the scale relativistic framework is implemented by passing to a fluid-like description (the fractality of space), considering the velocity field a fractal function explicitly depending on a scale variable (the fractal geometry of each geodesic) and defining two fractal velocity fields which are fractal functions of the scale variable $d t$ (the non-differentiability of space).

Consequently, replacing $d / d t$ with the fractal operator (42) and solving for both real and imaginary parts, (130) and (131) become, in a stationary isotropic case, taking the body force $X=0$ (constant gravitational field) and $\nabla \boldsymbol{U}=0$ (assuming a constant density of states for the "fluid particles" moving with the velocity $\boldsymbol{U}$ - see further in this section):

$$
\begin{array}{ll}
\boldsymbol{V} \cdot \nabla \boldsymbol{V}=-\frac{\nabla p}{\rho}+v \nabla^{2} \boldsymbol{V} & \mathrm{a} \\
\boldsymbol{U} \cdot \nabla \boldsymbol{V}+D \nabla^{2} \boldsymbol{V}=0 & \mathrm{~b}
\end{array}
$$

and 


$$
\begin{array}{ll}
\boldsymbol{V} \cdot \nabla \rho+\rho \nabla \cdot \boldsymbol{V}=0 & \mathrm{a} \\
\boldsymbol{U} \cdot \nabla \rho+D \nabla^{2} \rho=0 & \mathrm{~b}
\end{array}
$$

where $V$ represents the standard classical velocity, which does not depend on resolution, while the imaginary part, $U$, is a new quantity coming from resolution dependant fractal, $v=$ $\mu / \rho$ the kinematic viscosity and $D=\hbar / 2 m$ defines the amplitude of the fractal fluctuations.

The causes of the Casimir effect are described by quantum field theory, which states that all of the various fundamental fields, such as the electromagnetic field, must be quantized at each and every point in space. In a simplified view, a "field" in physics may be envisioned as if space were filled with interconnected vibrating balls and springs, and the strength of the field can be visualized as the displacement of a ball from its rest position. Vibrations in this field propagate and are governed by the appropriate wave equation for the particular field in question. The second quantization of quantum field theory requires that each such ballspring combination to be quantized, that is, that the strength of the field to be quantized at each point in space. Canonically, the field at each point in space is a simple harmonic oscillator, and its quantization places a quantum harmonic oscillator at each point. Excitations of the field correspond to the elementary particles of particle physics. However, even the vacuum has a vastly complex structure, so all calculations of quantum field theory must be made in relation to this model of vacuum. The vacuum has, implicitly, all of the properties that a particle may have: spin, or polarization in the case of light, energy, and so on. On average, all of these properties cancel out: the vacuum is, after all, "empty" in this sense. One important exception is the vacuum energy or the vacuum expectation value of the energy.

Let us consider here, vacuum, as a non-differentiable, Newtonian, 2D non-coherent quantum fluid whose entities (cvasi-particles) assimilate to vortex-type objects [38] (see Fig.9) and are described by the wave function $\Psi[39,40]$

$$
\Psi=c n(\underline{u} ; k)
$$

with

$$
\begin{array}{ll}
\underline{u}=\frac{K}{a} \underline{z}, & \mathrm{a} \\
\underline{z}=x+i y, & \mathrm{~b} \\
\frac{K}{a}=\frac{K^{\prime}}{b} & \mathrm{c} \\
K=\int_{0}^{\pi / 2}\left(1-k^{2} \sin ^{2} \phi\right)^{-1 / 2} d \phi, & \mathrm{d} \\
K^{\prime}=\int_{0}^{\pi / 2}\left(1-k^{\prime 2} \sin ^{2} \phi\right)^{-1 / 2} d \phi, & \mathrm{e} \\
k^{2}+k^{\prime 2}=1 & \mathrm{f}
\end{array}
$$


and $K, K^{\prime}$ complete elliptic integrals of the first kind of modulus $k$ [41], form a vortex lattice of constants $a, b$.

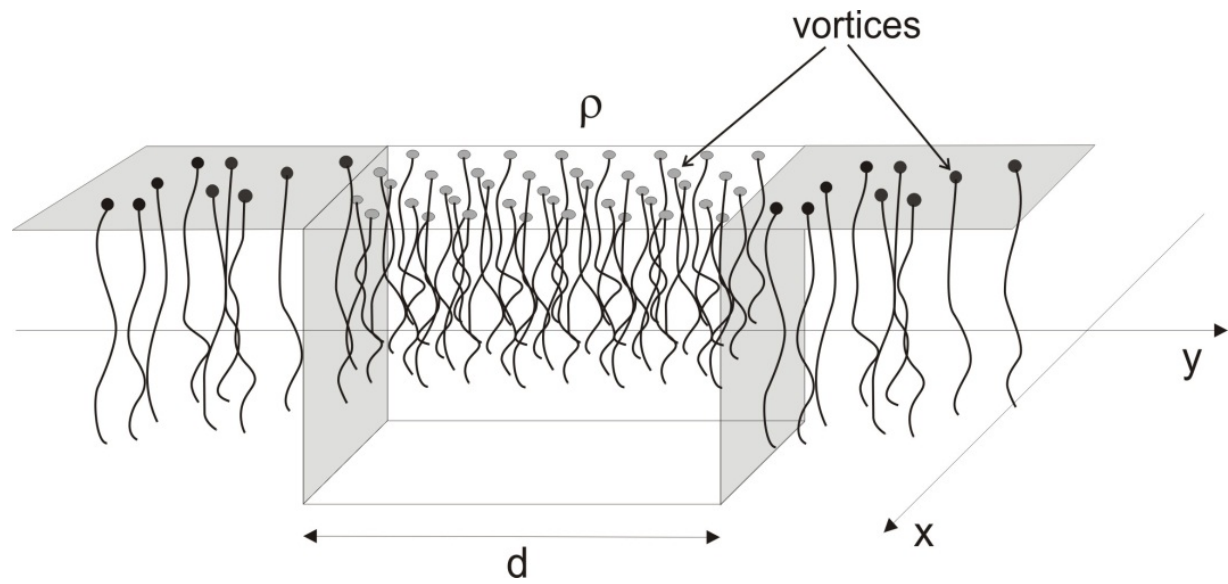

Figure 9. The vacuum from a Casimir cavity whose entities (cvasi-particles) are assimilated to vortex-type objects.

Applying in the complex plane [42], the formalism developed in [13] by means of the relation $\Psi=e^{F(z) / \Gamma}=c n(\underline{u} ; k)$ one introduces the complex potential

$$
F(\underline{z})=G(x, y)+i H(x, y)=\Gamma \ln [c n(\underline{u} ; k)]
$$

with $\Gamma$ the vortex constant. In the general case $\Gamma=c \Lambda=\hbar / m$ [38-40], the interaction scale being specified through $\Gamma$ 's value ( $\Lambda$ being considered as the Compton length).

Based on the complex potential (136), one defines the complex velocity field of the noncoherent quantum fluid, through the relation:

$$
v_{x}-i v_{y}=\frac{d F(\underline{z})}{d \underline{z}}=-\frac{\Gamma K}{a} \frac{s n(\underline{u} ; k) d n(\underline{u} ; k)}{c n(\underline{u} ; k)}
$$

or explicitly, using the notations [41, 42]: 


$$
\left.\begin{array}{cl}
s=s n(\alpha, k), & \mathrm{a} \\
c=c n(\alpha, k), & \mathrm{b} \\
d=d n(\alpha, k), & \mathrm{c} \\
\alpha=\frac{K}{a} x, & \mathrm{~d} \\
s_{1}=\operatorname{sn}\left(\beta, k^{\prime}\right), & \mathrm{e} \\
c_{1}=c n\left(\beta, k^{\prime}\right), & \mathrm{f} \\
d_{1}=d n\left(\beta, k^{\prime}\right), & \mathrm{g} \\
\beta & =\frac{K}{a} y \quad \mathrm{~h} \\
v_{x}-i v_{y}=-\Gamma \frac{K}{a}\left\{\frac{s c d\left[c_{1}^{2}\left(d_{1}^{2}+k^{2} c^{2} s_{1}^{2}\right)-s_{1}^{2} d_{1}^{2}\left(d^{2} c_{1}^{2}-k^{2} s^{2}\right)\right]}{\left(1-d^{2} s_{1}^{2}\right)\left(c^{2} c_{1}^{2}+s^{2} d^{2} s_{1}^{2} d_{1}^{2}\right)}\right. \\
+i \frac{s_{1} c_{1} d_{1}\left[c^{2}\left(d^{2} c_{1}^{2}-k^{2} s^{2}\right)+s^{2} d^{2}\left(d_{1}^{2}+k^{2} c^{2} s_{1}^{2}\right)\right]}{\left(1-d^{2} s_{1}^{2}\right)\left(c^{2} c_{1}^{2}+s^{2} d^{2} s_{1}^{2} d_{1}^{2}\right)}
\end{array}\right\}
$$

Having in view that $c n(\underline{u}+\underline{\Omega})=c n(\underline{u})$, where $\underline{\Omega}=2(2 m+1) K+2 n i K^{\prime}$ and $m, n= \pm 1, \pm 2 \ldots$, for $k \rightarrow$ 0 and $k^{\prime} \rightarrow 1$ limits, respectively, the quantum fluid, initially non-coherent (the amplitudes and phases of quantum fluid entities are independent) becomes coherent (the amplitudes and phases of quantum fluid entities are correlated [43]). In this context, from Fig. 10a,b of the equipotential curves $G\left(x_{r}, y_{r}\right)=$ const., for $k^{2}=0,1$, it results that the coherence of the quantum fluid reduces to its ordering in vortex streets - see Fig. III.2a for vortex streets aligned with the $O x$ axis and Fig. 10b for vortex streets aligned with the $O y$ axis. This process of ordering is achieved by generation of quasi-particles. Indeed, in the usual quantum mechanics the imaginary term $(i \Theta)$ from the energy, i.e. $E=E_{0}+i \Theta$, induces elementary excitations named resonances (for details see the collision theory [44]). Similarly, by extending the collision theory to the fractal space-time $[1,45]$, will imply that the presence of the imaginary term $H\left(x_{r}, y_{r}\right)$ in the potential $F(\underline{z})$ will generate quasi-particles, as well.

Now, writing the Navier-Stokes equation (132a) and the equation of continuity (133a) in scale relativity theory for constant density (incompressible fluids) in two dimensions, one gets

$$
\begin{array}{ll}
\frac{\partial p}{\partial x}=\rho \mathrm{D}\left(\frac{\partial^{2} v_{x}}{\partial x^{2}}+\frac{\partial^{2} v_{x}}{\partial y^{2}}\right)-\rho\left(v_{x} \frac{\partial v_{x}}{\partial x}+v_{y} \frac{\partial v_{x}}{\partial y}\right) & \mathrm{a} \\
\frac{\partial p}{\partial y}=\rho \mathrm{D}\left(\frac{\partial^{2} v_{y}}{\partial x^{2}}+\frac{\partial^{2} v_{y}}{\partial y^{2}}\right)-\rho\left(v_{x} \frac{\partial v_{y}}{\partial x}+v_{y} \frac{\partial v_{y}}{\partial y}\right) & \mathrm{b}
\end{array}
$$




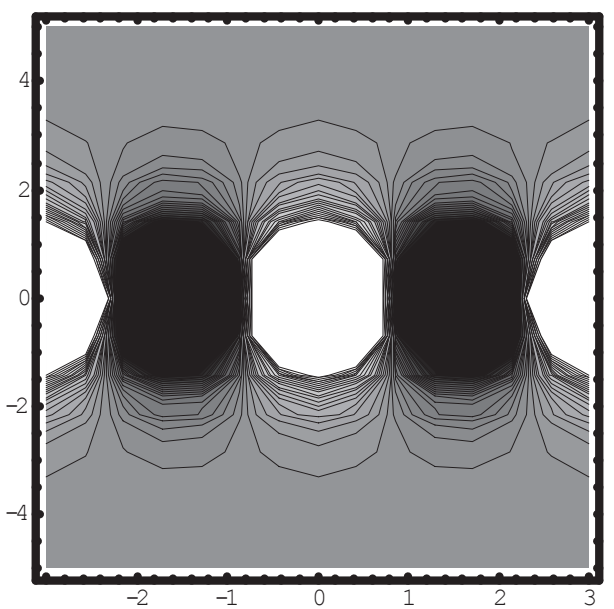

(a)

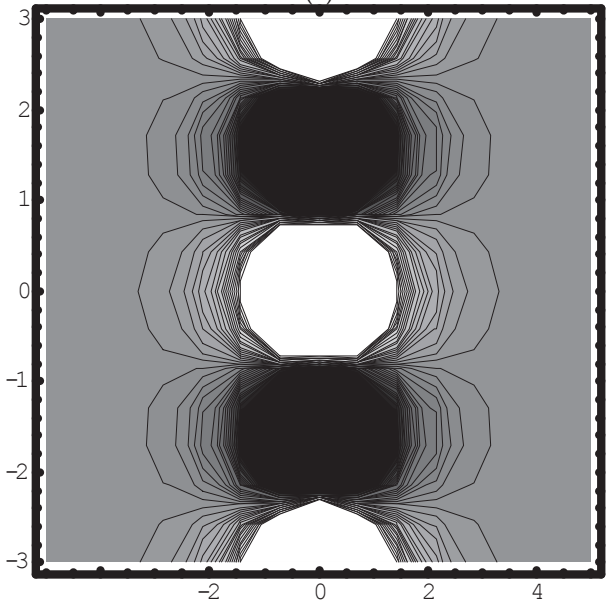

(b)

Figure 10. The equipotential curves $G\left(x_{r} y_{r}\right)=$ const., a) for vortex streets aligned with the $O x$ axis and b) for vortex streets aligned with the Oy axis.

$$
\frac{\partial v_{x}}{\partial x}+\frac{\partial v_{y}}{\partial y}=0
$$

where the shear viscosity $v$ is replaced by $D$ since we are dealing here with a non-differentiable quantum fluid.

Then, after some rather long yet elementary calculus one gets from $(140 a, b)$ through the degenerations : 
i.

$$
\begin{aligned}
k=0, \quad k^{\prime}=1, \quad K=\frac{\pi}{2}, \quad K^{\prime}=\infty & \\
& p_{y}(\alpha)=-p_{0} \sinh ^{2}\left(\frac{\pi d}{2 a}\right) \frac{1-\tan ^{2} \alpha}{\cos (2 \alpha)+\cosh \left(\frac{\pi d}{a}\right)} \\
& p_{x}(\beta)=-p_{0} \sin ^{2}\left(\frac{\pi d_{1}}{2 a}\right) \frac{1+\tanh ^{2} \beta}{\cos \left(\frac{\pi d_{1}}{a}\right)+\cosh (2 \beta)}
\end{aligned}
$$

with

$$
\begin{array}{ll}
p_{0}=\frac{\hbar^{2} \pi^{2} \rho}{4 M^{2} a^{2}} ; & \text { a } \\
\alpha=\frac{\pi x}{2 a} ; & \text { b } \\
\beta=\frac{\pi y}{2 a} & \text { c }
\end{array}
$$

and

ii.

$$
\begin{gathered}
k=1, \quad k^{\prime}=0, \quad K=\infty, \quad K^{\prime}=\frac{\pi}{2} \\
p_{y}\left(\alpha^{\prime}\right)=-p_{0}^{\prime} \sin ^{2}\left(\frac{\pi d}{2 b}\right) \frac{1+\tanh ^{2} \alpha^{\prime}}{\cos \left(\frac{\pi d}{b}\right)+\cosh \left(2 \alpha^{\prime}\right)} \\
p_{x}\left(\beta^{\prime}\right)=-p_{0}^{\prime} \sinh ^{2}\left(\frac{\pi d_{1}}{2 b}\right) \frac{1-\tan ^{2} \beta^{\prime}}{\cos \left(2 \beta^{\prime}\right)+\cosh \left(\frac{\pi d_{1}}{b}\right)}
\end{gathered}
$$

with

$$
\begin{array}{ll}
p_{0}^{\prime}=\frac{\hbar^{2} \pi^{2} \rho}{4 M^{2} b^{2}} ; & \mathrm{a} \\
\alpha^{\prime}=\frac{\pi x}{2 b} ; & \mathrm{b} \\
\beta^{\prime}=\frac{\pi y}{2 b} & \mathrm{c}
\end{array}
$$


Here, $\rho$ is the quantum fluid's density, $M$ the mass of the quantum fluid entities, $d$ and $d_{1}$ are the elementary space intervals considered along the $O y$ and $O x$ axis, respectively.

In other words, non-differentiability and coherence of the quantum fluid due to constraints, generate pressure along the $O x$ and $O y$ axis.

Moreover, one can show that the equation of continuity (141) is identically satisfied for both cases of degeneration.

Let us consider a Casimir cavity consisting of the vacuum with the vortex lattice depicted above and two metallic slabs, that constitute the plates of the cavity, placed at a distance $d$ apart, parallel to each other and to the $x \mathrm{Oz}$ plane (see Fig. III.1). According to the analysis from the previous section, one can see that if the quantum fluid is placed in a potential well with infinite walls (the case of the Casimir cavity analyzed here, where the two plates are the constraints of the quantum fluid), along a direction perpendicular to the walls (the Oy axis here) a coherent structure, a vortex street forms (see Fig. III.2b). Consequently, by integrating (144a,b) with (145a-c) over $\alpha_{r}$ and $\beta_{r}$, and using the result in the quantization rule:

$$
\int_{d_{1}}^{d_{2}} k d x=n \pi, \quad n=1,2,3, \ldots
$$

where $d_{1} \sim m \pi a, d \sim n \pi b$, with $m, n=1,2, \ldots$. , one gets

$$
\begin{aligned}
& \frac{\pi}{2} \frac{p_{y}}{p_{0}^{\prime}}=2 r \arctan \left[\tan \left(\frac{n \pi^{2}}{2}\right) \tanh \left(\frac{m \pi^{2}}{4 r}\right)\right] \tan ^{-1}\left(n \pi^{2}\right)-r \tanh \left(\frac{m \pi^{2}}{4 r}\right) \\
& \frac{\pi}{2} \frac{p_{x}}{p_{0}^{\prime}}=-2 \arctan \left[\tan \left(\frac{n \pi^{2}}{4}\right) \tanh \left(\frac{m \pi^{2}}{2 r}\right)\right] \tanh ^{-1}\left(\frac{m \pi^{2}}{r}\right)+\tan \left(\frac{n \pi^{2}}{4}\right)
\end{aligned}
$$

where

$$
\begin{array}{ll}
p_{0}^{\prime}=\frac{\hbar^{2} \pi^{2} \rho}{4 M^{2} b^{2}} ; & \mathrm{a} \\
\alpha_{r}=\frac{\pi}{2} \frac{x}{a} ; & \mathrm{b} \\
\beta_{r}=\frac{\pi}{2} \frac{y}{b} ; & \mathrm{c} \\
r=\frac{b}{a} & \mathrm{~d}
\end{array}
$$

Graphically this is presented in Fig. III.3a,b for different values of the parameters $m, n=1,2, \ldots$. and $r$. 
If the plates were in the $y O z$ plane the constraints being along the $O x$ axis, vortex streets would form along this axis and the result in $(142 a, b)$ with $(143 a-c)$ would have been applied, i.e. the cases i) or ii) are identical, yet they depend on the geometry chosen.

Firstly, one can notice that the pressure $p_{y}$ on the plates, given by (147a), stabilizes for great $r$ values, is always negative and an attractive force results (see Fig. $11 \mathrm{a}$ ), as is the case of the Casimir force (128).

Secondly, the theory predicts, that besides the pressure $p_{y}$ acting on the plates, there must be yet another pressure, $p_{x}$ (see Fig. $11 \mathrm{~b}$ ), acting along the $O x$ axis and given by $(147 \mathrm{~b})$. One can see that this pressure annuls for great $r$ values, and has a minimum for some values of the parameters $m, n$. This result is new and should be checked by experiments.

Moreover, if one tries to compute the order of magnitude of this force, and replaces in (144a) : $\hbar=1.05410^{-34} \mathrm{~J} . \mathrm{s}, m=9.110^{-31} \mathrm{~kg}, \rho \sim 10^{21} \mathrm{~cm}^{-3}, b=1 \AA \AA$ (values specific to a bosonic gas, i.e. found in high-Tc superconductors [46]) and $d \sim 5 b$ (the distance between the plates), gets a value for $p_{y} \cong 6.1810^{10} \mathrm{~N} \mathrm{~m}^{-2}$ the same order of magnitude as the value calculated using (128), $F_{C} \cong 2.08$ $10^{10} \mathrm{~N} \mathrm{~m}^{-2}$.

As a final test, let us study the case of a Casimir cavity, as a rectangle of sides $d_{1}, d$. Now, the plates induce constraints along both $O x$ and $O y$ axis, thus correlations (vortex streets) form along these directions and one should use the degenerations i) and ii), simultaneously. Consequently, from $(142 a, b)$ with $(143 a-c)$ and $(144 a, b)$ with $(145 a-c)$ one gets

$$
p_{y \text { rect }}\left(\alpha, \alpha^{\prime}\right)=-\frac{\hbar^{2} \pi^{2} \rho}{4 m^{2}}\left(\frac{1}{a^{2}} \frac{\sinh ^{2} A}{\cos ^{2} \alpha}\left(1+\frac{\cosh 2 A}{\cos 2 \alpha}\right)^{-1}+\frac{1}{b^{2}} \frac{\sin ^{2} B}{\cosh ^{2} \alpha^{\prime}}\left(1+\frac{\cos 2 B}{\cosh 2 \alpha^{\prime}}\right)^{-1}\right)
$$

with

$$
A=\frac{\pi d}{2 a} ; \quad B=\frac{\pi d}{2 b}
$$

and

$$
p_{\text {x rect }}\left(\beta, \beta^{\prime}\right)=-\frac{\hbar^{2} \pi^{2} \rho}{4 m^{2}}\left(\frac{1}{a^{2}} \frac{\sin ^{2} A^{\prime}}{\cosh ^{2} \beta}\left(1+\frac{\cos 2 A^{\prime}}{\cosh 2 \beta}\right)^{-1}+\frac{1}{b^{2}} \frac{\sinh ^{2} B^{\prime}}{\cos ^{2} \beta^{\prime}}\left(1+\frac{\cosh 2 B^{\prime}}{\cos 2 \beta^{\prime}}\right)^{-1}\right)
$$

with

$$
A^{\prime}=\frac{\pi d_{1}}{2 a} ; \quad B^{\prime}=\frac{\pi d_{1}}{2 b}
$$



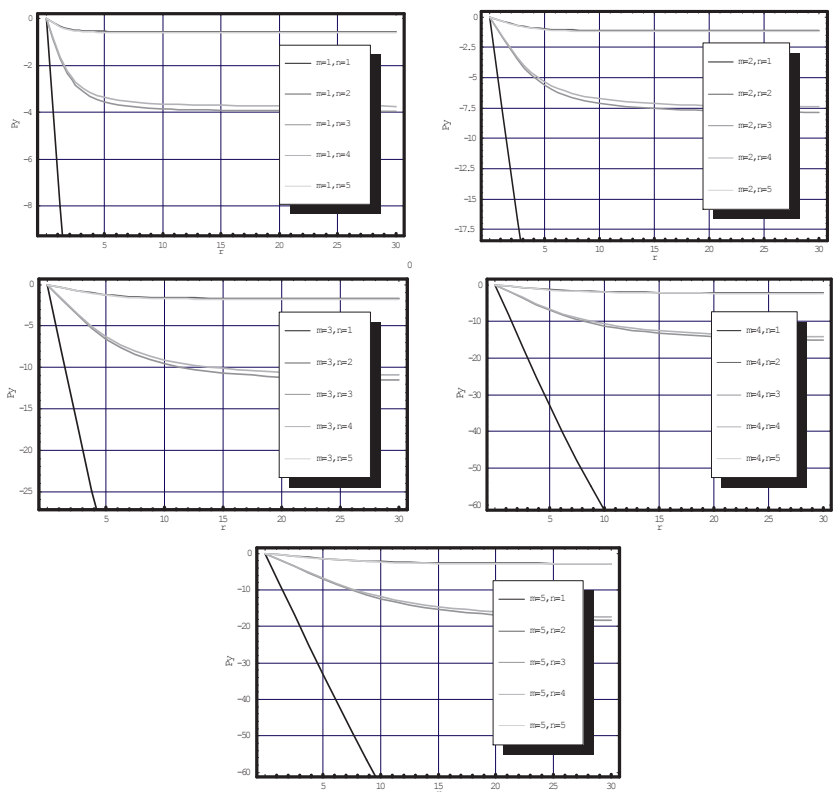

(a)
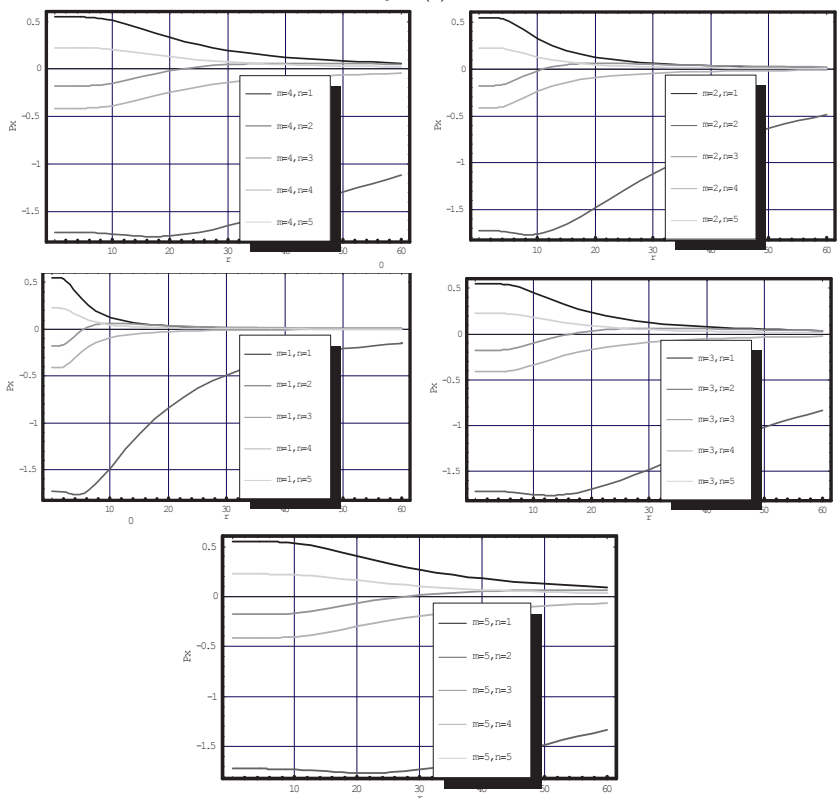

(b)

Figure 11. a) Plot of the pressure $p_{y}$ on the plates, versus the parameter $r$ for different values of parameters $m, n$; b) Plot of the pressure $p_{x}$ versus the parameter $r$ for different values of parameters $m, n$. 
At every point $(x, y)$ there is a pressure formed of the two constraints. Consequently, adding the pressures in (149) and (151) and using again the result in (146) (i.e. $d_{1} \sim m \pi a, d \sim n \pi b$, where $m, n=1,2, \ldots$.$) one gets:$

$$
\begin{aligned}
& -\frac{p_{r e c t}\left(\alpha_{r}, \beta_{r}\right)}{p_{0}^{\prime}}=r^{2} \frac{\sinh ^{2}\left(\frac{n \pi^{2}}{2} r\right)}{\cos ^{2} \alpha_{r}}\left(1+\frac{\cosh \left(n \pi^{2} r\right)}{\cos \left(2 \alpha_{r}\right)}\right)^{-1}+\frac{\sin ^{2}\left(\frac{n \pi^{2}}{2}\right)}{\cosh ^{2}\left(\frac{\alpha_{r}}{r}\right)}\left(1+\frac{\cos \left(n \pi^{2}\right)}{\left.\cosh \left(\frac{2 \alpha_{r}}{r}\right)\right)^{-1}+}\right. \\
& +r^{2} \frac{\sin ^{2}\left(\frac{m \pi^{2}}{2}\right)}{\cosh ^{2}\left(r \beta_{r}\right)}\left(1+\frac{\cos \left(m \pi^{2}\right)}{\cosh \left(2 r \beta_{r}\right)}\right)^{-1}+\frac{\sinh ^{2}\left(\frac{1}{r} \frac{m \pi^{2}}{2}\right)}{\cos ^{2} \beta_{r}}\left(1+\frac{\cosh \left(\frac{m \pi^{2}}{r}\right)}{\cos \left(2 \beta_{r}\right)}\right)^{-1}
\end{aligned}
$$

where

$$
p_{0}^{\prime}=\frac{\hbar^{2} \pi^{2} \rho}{4 M^{2} b^{2}} ; \quad \alpha_{\mathrm{r}}=\frac{\pi}{2} \frac{x}{a}=\frac{\pi}{2} x_{r} ; \quad \beta_{\mathrm{r}}=\frac{\pi}{2} \frac{y}{b}=\frac{\pi}{2} y_{r} ; \quad r=\frac{b}{a}
$$

Furthermore, we integrate (153) over $x_{r}$ and $y_{r}$, respectively, in order to find a value of the pressure acting on the sides of the rectangular enclosure. After some long, yet elementary calculus, one finds:

$$
\begin{gathered}
\frac{p_{\text {rect }}}{p_{0}^{\prime}}=-4 n r^{2} \frac{\operatorname{arctg}\left[\operatorname{tg}\left(\frac{m \pi^{2}}{4}\right) \operatorname{th}\left(\frac{n \pi^{2}}{2} r\right)\right]}{\operatorname{th}\left(n \pi^{2} r\right)}+4 n r \frac{\operatorname{arctg}\left[\operatorname{tg}\left(\frac{n \pi^{2}}{2}\right) \operatorname{th}\left(\frac{m \pi^{2}}{4} \frac{1}{r}\right)\right]}{\operatorname{tg}\left(n \pi^{2}\right)}+ \\
+2 n r^{2} \operatorname{tg}\left(\frac{m \pi^{2}}{4}\right)-2 n r \operatorname{th}\left(\frac{m \pi^{2}}{4} \frac{1}{r}\right)- \\
-4 m \frac{\operatorname{arctg}\left[\operatorname{tg}\left(\frac{n \pi^{2}}{4}\right) \operatorname{th}\left(\frac{m \pi^{2}}{2} \frac{1}{r}\right)\right]}{\operatorname{th}\left(m \pi^{2} \frac{1}{r}\right)}+4 m r \frac{\operatorname{arctg}\left[\operatorname{tg}\left(\frac{m \pi^{2}}{2}\right) \operatorname{th}\left(\frac{n \pi^{2}}{4} r\right)\right]}{\operatorname{tg}\left(m \pi^{2}\right)}+ \\
+2 m \operatorname{tg}\left(\frac{n \pi^{2}}{4}\right)-2 m r \operatorname{th}\left(\frac{n \pi^{2}}{4} r\right)
\end{gathered}
$$

Plots of (155) for various values of parameters $m, n=1,2, \ldots$ and $r$ are depicted in Fig. III.4a,b. 

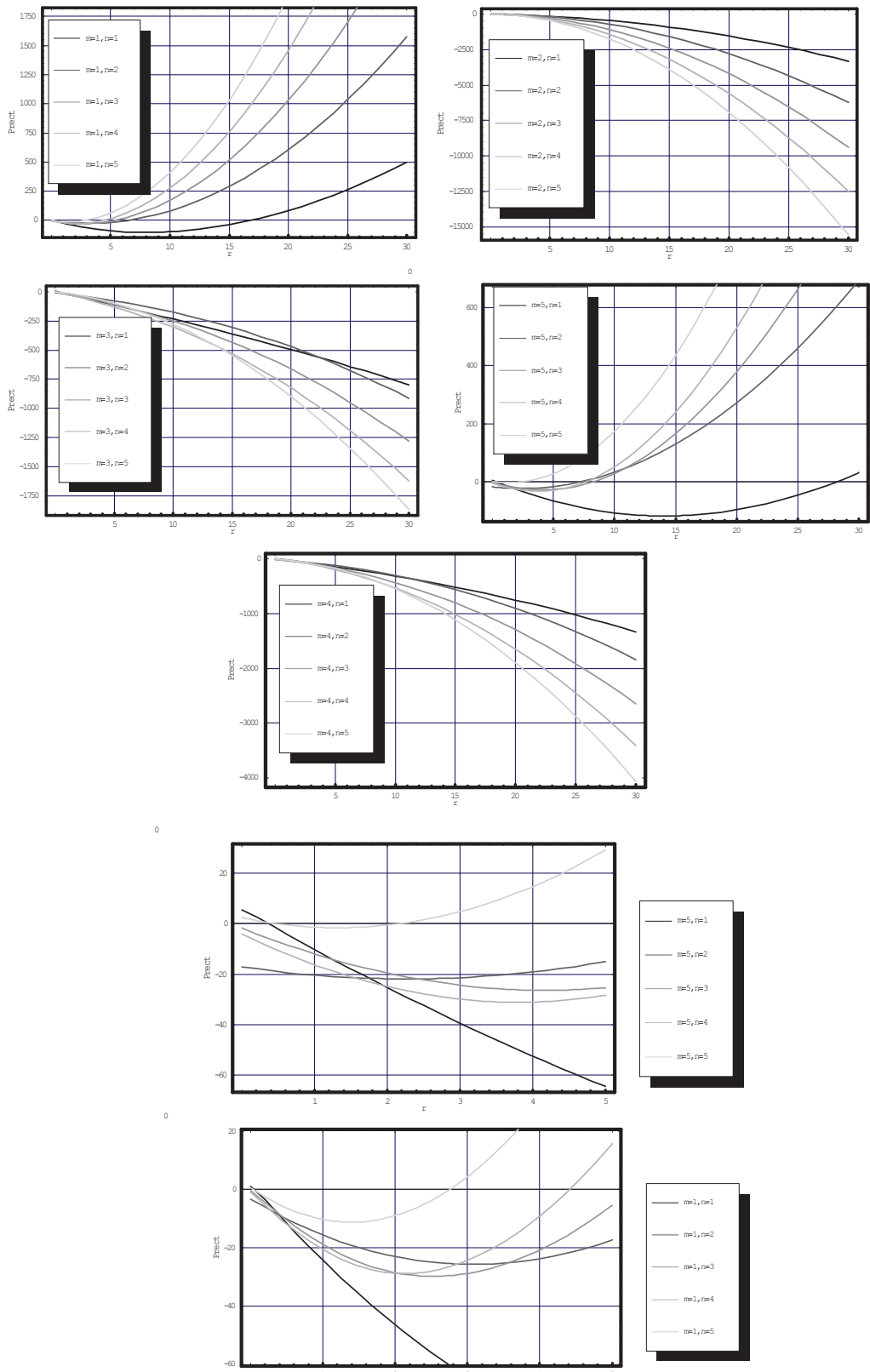

Figure 12. a) Plots of $p_{\text {rect }}$ versus the parameter $r$ for various values of parameters $m, n=1,2, \ldots ; b$ ) the same plot, yet we present here a magnification of the domain of $r$ for highly asymmetric values of $m, n(1,5$ and 5,1$)$. 
One can notice that if the two parameters $m$ and $n$ have close values, the force acting on the Casimir rectangle is always negative and decreases exponentially for increasing $r$. For parameters $m$ and $n(1,5$ and 5,1, i.e. very asymmetric) the force has negative and positive domains (see Fig. 12 b) and increases exponentially for increasing $r$. Moreover, if one tries to find the positive and negative domains, and solve (155) for $m=5, n=5$ finds $p_{\text {rect }}<0$ for $0.45753 \leq r \leq 2.18565$ and $p_{\text {rect }}>0$ for $r>2.18565$ and $r<0.45753$. This result is in agreement with the calculus of regularization using the Abel-Plana formula where $E<0$ for $0.36537 \leq L / l \leq$ 2.73686 and $E>0$ for $L / l>2.73686$ and $L / l<0.36537$ [47].

\section{Fractal approximation of motion in mass transfer: release of drug from polimeric matrices}

Polymer matrices can be produced in one of the following forms: micro/nano-particles, micro/ nano capsules, hydro gels, films, patches.Our new approach considers the entire system (drug loaded polymer matrix in the release environment) as a type of "fluid" totally lacking interaction or neglecting physical interactions among particles. At the same time, the induced complexity is replaced by fractality. This will lead to particles moving on certain trajectories called geodesics within fractal space. This assumption represents the basis of the fractal approximation of motion in Scale Relativity Theory (SRT) [1, 2], leading to a generalized fractal "diffusion" equation that can be analyzed in terms of two approximations (dissipative and dispersive).

\subsection{The dissipative approximation}

In the dissipative approximation the fractal operator (42) takes the form $[48,49]$ :

$$
\frac{\hat{\partial}}{\partial t}=\frac{\partial}{\partial t}+\hat{\boldsymbol{V}} \cdot \nabla-i \mathrm{D}(d t)^{\left(2 / D_{F}\right)-1} \Delta
$$

As a consequence, we are now able to write the fractal "diffusion" type equation in its covariant form:

$$
\frac{\partial Q}{d t}=\frac{\partial Q}{\partial t}+(\hat{\boldsymbol{V}} \cdot \nabla) Q-i D(d t)^{\left(2 / D_{F}\right)-1} \Delta Q=0
$$

Separating the real and imaginary parts in (157), i.e.

$$
\frac{\partial Q}{\partial t}+V \cdot \nabla Q=0
$$




$$
-\boldsymbol{U} \cdot \nabla Q=\mathrm{D}(d t)^{\left(2 / D_{F}\right)-1} \Delta Q
$$

we can add these two equations and obtain a generalized "diffusion" type law in the form:

$$
\frac{\partial Q}{\partial t}+(\boldsymbol{V}-\boldsymbol{U}) \cdot \nabla Q=\mathrm{D}(d t)^{\left(2 / D_{F}\right)-1} \Delta Q
$$

\subsubsection{Standard "diffusion" type equation. Fick type law}

The standard "diffusion" law, i.e.:

$$
\frac{\partial Q}{\partial t}=\mathrm{D} \Delta Q
$$

results from (159) on the following assertions:

i. the diffusion path are the fractal curves of Peano's type. This means that the fractal dimension of the fractal curves is $D_{F}=2$.

ii. the movements at differentiable and non-differentiable scales are synchronous, i.e. $V=U$;

iii. the structure coefficient $\mathrm{D}$, proper to the fractal-nonfractal transition, is identified with the diffusion coefficient, i.e.

$D \equiv D$.

\subsubsection{Anomalous "diffusion" type equation. Weibull relation}

The anomalous diffusion law results from (IV.4) on the following assumptions:

i. $\quad$ the diffusion path are fractal curves with fractal dimension $D_{F} \neq 2$;

ii. the time resolution, $\delta t$, is identified with the differential element $d t$, i.e. the substitution principle can be applied also, in this case;

iii. the movements at differentiable and non-differentiable scales are synchronous, i.e. $V=U$.

Then, the equation (IV.4) can be written:

$$
\frac{\partial Q}{\partial t}=\mathrm{D}(d t)^{\left(2 / D_{F}\right)-1} \Delta Q
$$

In one-dimensional case, applying the variable separation method [50] 


$$
Q(t, x)=T(t) \cdot X(\boldsymbol{x})
$$

with the standard initial and boundary conditions:

$$
Q(t, 0)=0, Q(t, L)=0, Q(0, x)=F(x), 0 \leq x \leq L
$$

implies:

$$
\frac{1}{\mathrm{D}(d t)^{\left(2 / D_{F}\right)-1}} \frac{1}{T(t)} \frac{d T(t)}{d t}=\frac{1}{X(x)} \frac{d^{2} X(x)}{d x^{2}}=-m^{2}=-\left(\frac{n \pi}{L}\right)^{2}, n=1,2
$$

where $L$ is a system characteristic length, $m$ a separation constant, dependent on diffusion order $n$.

Accepting the viability of the substitution principle, from (164), through integration, results:

$$
\ln T=-m^{2} \mathrm{D} \int(d t)^{2 / D_{F}}
$$

Taking into consideration some results of the fractional integro-differential calculus [51, 52], (165) becomes:

$$
\begin{aligned}
& \ln T=-\frac{m^{2} \mathrm{D}}{\Gamma\left(\frac{2}{D_{F}}+1\right)} t^{\frac{2}{D_{F}}}, \quad \mathrm{a} \\
& \Gamma\left(\frac{2}{D_{F}}\right)=\int_{0}^{\infty} x^{\left(\frac{2}{D_{F}}\right)-1} e^{-x} d x
\end{aligned}
$$

Moreover, $(166 a, b)$ can be written under the form:

$$
T(t)=\exp \left[-\frac{m^{2} \mathrm{D}}{\Gamma\left(\frac{2}{D_{F}}+1\right)} t^{\frac{2}{D_{F}}}\right]
$$

The relative variation of concentrations, time dependent, is defined as: 


$$
T(t)=\frac{Q_{\infty}-Q_{t}}{Q_{\infty}}
$$

where $Q_{t}$ and $Q_{\infty}$ are cumulative amounts of drug released at time $t$ and infinite time.

From (167) and (168) results:

$$
\frac{Q_{t}}{Q_{\infty}}=1-\exp \left[-\frac{m^{2} \mathrm{D}}{\Gamma\left(\frac{2}{D_{F}}+1\right)} t^{\frac{2}{D_{F}}}\right]
$$

equation similar to Weibull relation $\frac{Q_{t}}{Q_{\infty}}=1-\exp \left(-a t^{b}\right), a$ and $b$ representing constants specific for each system that are defined by:

$$
\begin{array}{ll}
a=\frac{m^{2} D}{\Gamma\left(\frac{2}{D_{F}}+1\right)}=\left(\frac{n \pi}{L}\right)^{2} \frac{D}{\Gamma\left(\frac{2}{D_{F}}+1\right)} & \mathrm{a} \\
b=\frac{2}{D_{F}} & \mathrm{~b}
\end{array}
$$

We observe that both constants, $a$ and $b$, are functions of the fractal dimension of the curves on which drug release mechanism take place, dimension that is a measure of the complexity and nonlinear dynamics of the system. Moreover, constant $a$ depends, also, on the "diffusion" order $n$.

\subsubsection{The correspondence between theoretical model and experimental results}

The experimental and Weibull curves for HS (starch based hydrogels loaded with levofloxacin) and GA (GEL-PVA microparticles loaded with chloramphenicol) samples are plotted in Fig. 13.

The experimental data allowed to determine the values of Weibull parameters ( $a$ and $b$ ), and implicitly, the value of the fractal dimension from the curve on which release takes place [55].

These values confirmed that the complexity of the phenomena determines, also, naturally, a complex trajectory for the drug particles. Most values are between 1 and 3, in agreement with the values usually accepted for fractal process; higher values denotes the fact that, either fractal dimension must be redefined as function of structure "classes", or the drug release process is complex, involving many freedom degrees in the phase space [56]. Another observation that can be made based on this results is that the samples with $D_{F}<2$ manifests a "sub-diffusion" 

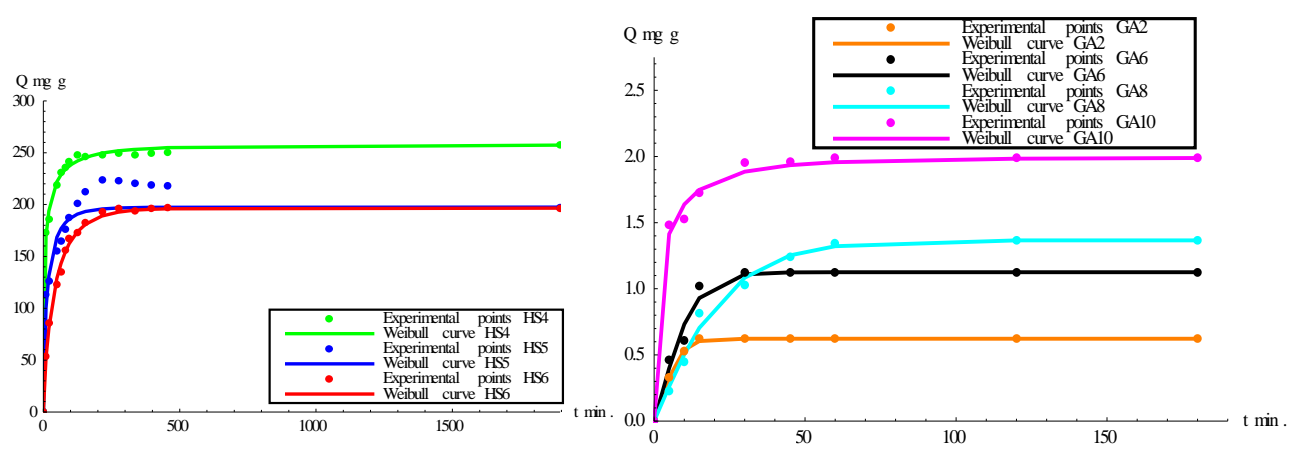

Figure 13. Experimental and Weibull curves for HS (left plot) and GA samples (right plot).

and, in the other, with $D_{F}>2$, the release process is of super-diffusion, classification in concordance with the experimental observation that this samples exhibit a "faster" diffusion, with a higher diffusion rate, in respect with the other samples [55].

\subsection{The dispersive approximation}

Let us now consider that, in comparison with dissipative processes, convective and dispersive processes are dominant ones. In these conditions, the fractal operator (42) takes the form:

$$
\frac{\hat{\partial}}{d t}=\frac{\partial}{\partial t}+(\hat{\boldsymbol{V}} \cdot \nabla)+\frac{\sqrt{2}}{3} \mathrm{D}^{3 / 2}(d t)^{\left(3 / D_{F D}\right)-1} \nabla^{3}
$$

Consequently, we are now able to write the diffusion equation in its covariant form, as a Korteweg de Vries type equation:

$$
\frac{\partial Q}{d t}=\frac{\partial Q}{\partial t}+(\hat{V} \cdot \nabla) Q+\frac{\sqrt{2}}{3} D^{3 / 2}(d t)^{\left(3 / D_{F D}\right)-1} \nabla^{3} Q=0
$$

If we separate the real and imaginary parts from Eq. (172), we shall obtain:

$$
\begin{array}{ll}
\frac{\partial Q}{\partial t}+\boldsymbol{V} \cdot \nabla Q+\frac{\sqrt{2}}{3} D^{3 / 2}(d t)^{\left(3 / D_{F}\right)-1} \nabla^{3} Q=0 & \text { a } \\
-\boldsymbol{U} \cdot \nabla Q=0 & \mathrm{~b}
\end{array}
$$

By adding them, the fractal diffusion equation is: 


$$
\frac{\partial Q}{\partial t}+(\boldsymbol{V}-\boldsymbol{U}) \cdot \nabla Q+\frac{\sqrt{2}}{3} \boldsymbol{D}^{3 / 2}(d t)^{\left(3 / D_{F}\right)-1} \nabla^{3} Q=0
$$

From Eq. (173b) we see that, at fractal scale, there will be no $Q$ field gradient.

Assuming that $|\boldsymbol{V}-\boldsymbol{U}|=\sigma \cdot Q$ with $\sigma=$ constant (in systems with self structuring processes, the speed fluctuations induced by fractal - non fractal are proportional with the concentration field [55]), in the particular one-dimensional case, equation (174) with normalized parameters:

$$
\begin{array}{ll}
\bar{\tau}=\omega t, & \mathrm{a} \\
\bar{\xi}=k x, & \mathrm{~b} \\
\Phi=\frac{Q}{Q_{0}} & \mathrm{c}
\end{array}
$$

and normalizing conditions:

$$
\frac{\sigma Q_{0} k}{6 \omega}=\frac{\sqrt{2}}{3} \frac{\mathrm{D}^{3 / 2}(d t)^{\left(3 / D_{F}\right)-1} k^{3}}{\omega}=1
$$

take the form:

$$
\partial_{\bar{\tau}} \phi+6 \phi \partial_{\bar{\xi}} \phi+\partial_{\bar{\xi} \bar{\xi} \bar{\xi}} \phi=0
$$

In relations (175a,b,c) and (176) $\omega$ corresponds to a characteristic pulsation, $k$ to the inverse of a characteristic length and $Q_{0}$ to balanced concentration.

Through substitutions:

$$
\begin{array}{ll}
w(\theta)=\phi(\bar{\tau}, \bar{\xi}), & \mathrm{a} \\
\theta=\bar{\xi}-u \bar{\tau} & \mathrm{b}
\end{array}
$$

eq.(177), by double integration, becomes:

$$
\frac{1}{2} w^{\prime 2}=F(w)=-\left(w^{3}-\frac{u}{2} w^{2}-g w-h\right)
$$


with $g, h$ two integration constants and $u$ the normalized phase velocity. If $F(w)$ has real roots, equation (177) has the stationary solution:

$$
\phi(\bar{\xi}, \bar{\tau}, s)=2 a\left(\frac{E(s)}{K(s)}-1\right)+2 a \cdot c n^{2}\left[\frac{\sqrt{a}}{s}\left(\bar{\xi}-\frac{\bar{u}}{2} \bar{\tau}+\overline{\xi_{0}}\right) ; s\right]
$$

where $c n$ is Jacobi's elliptic function of $s$ modulus [41], $a$ is the amplitude, $\bar{\xi}_{0}$ is a constant of integration and

$$
\begin{aligned}
& K(s)=\int_{0}^{\pi / 2}\left(1-s^{2} \sin ^{2} \phi\right)^{-1 / 2} d \phi \quad \text { a } \\
& E(s)=\int_{0}^{\pi / 2}\left(1-s^{2} \sin ^{2} \phi\right)^{1 / 2} d \phi \quad b
\end{aligned}
$$

are the complete elliptic integrals [41].

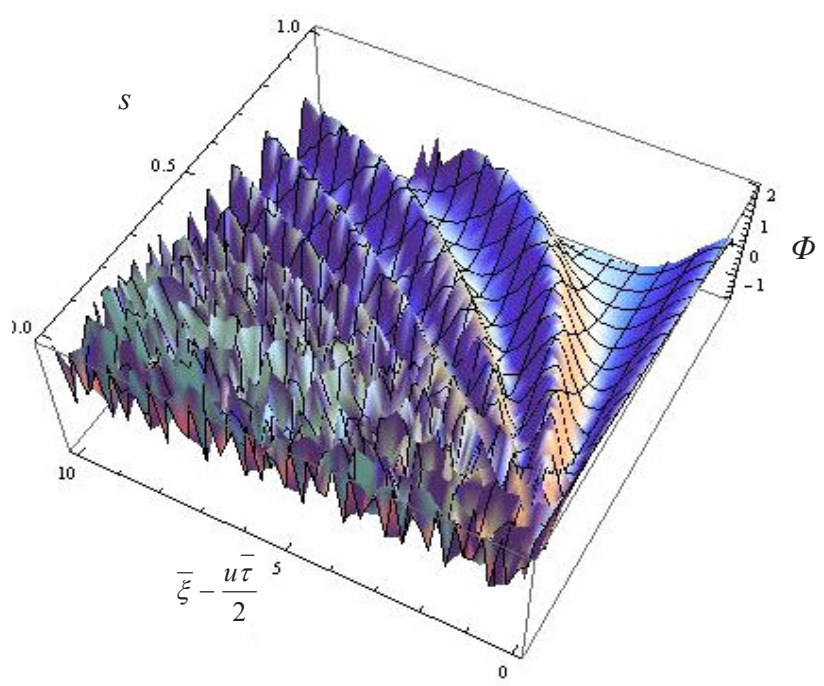

Figure 14. One-dimensional cnoidal oscillation modes of the field $\Phi$ 
Parameter $s$ represents measure characterizing the degree of nonlinearity in the system. Therefore, the solution (180) contains (as subsequences for $s=0$ ) one-dimensional harmonic waves, while for $s \rightarrow 0$ one-dimensional wave packet. These two subsequences define the nonquasi-autonomous regime of the drug release process $[48,49,55]$, i.e. the system should receive external energy in order to develop. For $s=1$, the solution (180) becomes one-dimensional soliton, while for $s \rightarrow 1$, one-dimensional soliton packet will be generated. The last two imply a quasi-autonomous regime (self evolving and independent [48]) for drug particle release process $[48,49,55]$.

The three dimensional plot of solution (180) shows one-dimensional cnoidal oscillation modes of the concentration field, generated by similar trajectories of the drug particles (see Fig. 14). We mention that cnoidal oscillations are nonlinear ones, being described by the elliptic function $c n$, hence the name (cnoidal).

It is known that in nonlinear dynamics, cnoidal oscillation modes are associated with nonlinear lattice of oscillators (the Toda lattice [56]). Consequently, large time scale drug particle ensembles can be compared to a lattice of nonlinear oscillators which facilitates drug release process.

\subsubsection{The correspondence between theoretical model and experimental results}

In what follows we identify the field $\Phi$ from relation (180) with normalized concentration field of the released drug from micro particles.

For best correlation between experimental data and the theoretical model (for each sample) we used a planar intersection of the graph in Fig. 14 [57], in order to obtain two-dimensional plots.

The highest value of the correlation coefficient (for two data sets: one obtained from the planar intersection, the other from experimental data) will represent the best approximation of experimental data with the theoretical model.

Our goal was to find the right correlation coefficient which should be higher than 0.6-0.7, in order to demonstrate the relevance of the model we had in view. Figs. 15 show experimental and theoretical curves that were obtained through this method, where $R^{2}$ represents the correlation coefficient and $\eta$ a normalized variable which is simultaneously dependent on normalized time and on nonlinear degree of the system (s parameter). Geometrically, $\eta$ represents the congruent angle formed by the time axis and the vertical intersection plane. 

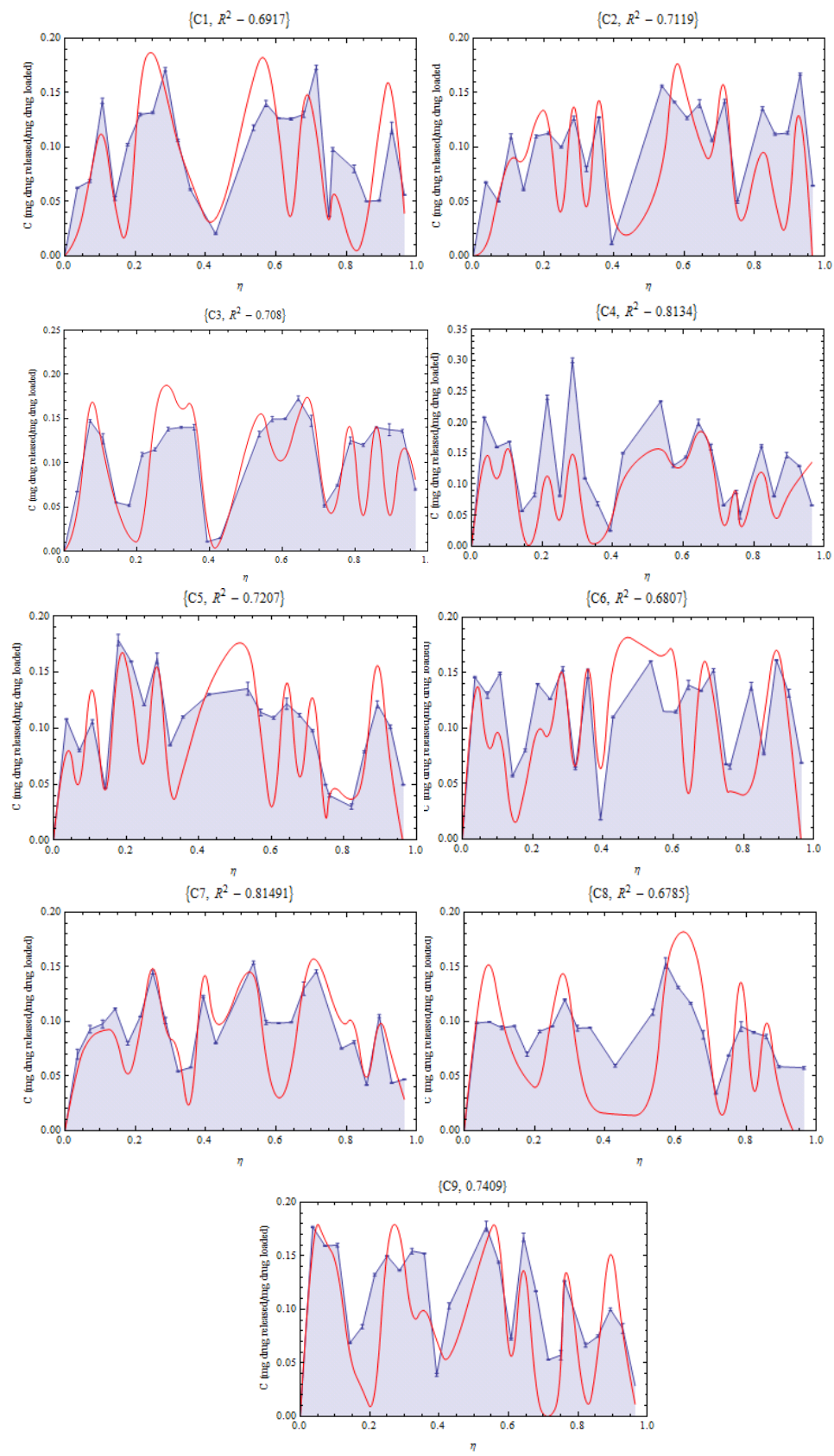

Figure 15. The best correlations among experimental and theoretical curves (blue line - experimental curve, red line theoretical curve). 


\section{Conclusions}

i. Scale relativistic framework is implemented by passing to a fluid-like description (the fractality of space), considering the velocity field a fractal function explicitly depending on a scale variable (the fractal geometry of each geodesic) and defining two fractal velocity fields which are fractal functions of the scale variable $d t$ (the non-differentiability of space).

An application of these principles to the motion equation of free particles leads to the occurence of a supplementary TISE (time independent, Schrödinger-type equation) and the following interesting results :

- $\zeta(x)$ behaves like a wave function on small distances (the same magnitude as the Compton length);

- for $\gamma(x)$ a velocity potential well, $U(x)$ is quantified;

- for the harmonic oscillator case, the limit velocity $\chi$ has discrete values, and only the first value is less than the velocity of light, $c$;

- in the double-well velocity potential, the complex velocity $U(x)$ is again quantized, this time the levels are equally spaced at a value of $\hbar \ln 2$;

- if one takes $\phi_{R}=\phi_{L}=\pi / 2$, singularities are obtained for $x-x_{0}=\Lambda / 2$ and for $x-x_{0}=$ $\Lambda / 4$ one gets minima for $U(x)=c$ in a double-well velocity potential;

- since we considered here the one-dimensional case we get the solution of a single row of rectilinear vortices, which has already been referred to as characterizing a surface of discontinuity;

- a typical bound state in a double-well has two classically allowed regions, where the velocity potential is less than the limit velocity; these regions are separated by a classically forbidden region, or barrier, where the velocity potential is larger than the limit velocity;

- for tunneling case, there is a nonzero transmission, reflection coefficient, which leads to the proof of the transport of the $V$ field by the motion of the Newtonian fluid with velocity $U(x)$, on small distances (of the order of magnitude of Compton length).

ii. We analyzed vacuum from the Casimir cavity, considered a non-differentiable, Newtonian, 2D non-coherent quantum fluid, by writing the Navier-Stokes equations inscale relativity theory's framework. As a result thefollowing resultsmaybeextracted:

- the (vector) velocity field $V$ and/or the (scalar) density field $\rho$ behave like a wave function on small distances (the same magnitude as the Compton length);

- the (vector) velocity field $V$ and/or the (scalar) density field $\rho$ are transported by the motion of the Newtonian fluid with velocity $U$, on small distances (the same magnitude as the Compton length);

Also, the entities assimilated to vortex-type objects from the Casimir cavity, initially non-coherent, become coherent due to constraints induced by the presence of walls and generate pressure along the $O x$ and $O y$ axis, thus one can stress out : 
- the pressure $p_{y}$ on the plates, is negative and an attractive force results, as is the case of the Casimir force;

- besides the pressure $p_{y}$ acting on the plates, there must be yet another pressure, $p_{x}$ acting along the $O x$ axis;

- the order of magnitude of this force, $p_{y} \cong 6.1810^{10} \mathrm{~N} \mathrm{~m}^{-2}$ is the same with the value of the classical Casimir force calculation, $F_{C} \cong 2.0810^{10} \mathrm{~N} \mathrm{~m}^{-2}$;

- in the case of the Casimir cavity from inside a rectangular enclosure of sides $d_{1}, d$, the plates induce constraints along both $O x$ and $O y$ axis, and one can notice that if the two parameters $m$ and $n$ have close values, the force acting on the Casimir rectangle is always negative and for parameters $m$ and $n$ very asymmetric the force has negative and positive domains, in agreement with the calculus of regularization using the Abel-Plana formula.

iii. Using fractional calculus, the fractal "diffusion" equation give rise to Weibull relation, a statistical distribution function of wide applicability, inclusively in drug release studies. In this approach, we consider all the simultaneous phenomena involved, equivalent with complexity and fractality, offering, in this way, a physical base to this equation and for its parameters. They are functions of fractal dimension of the curves on which drug release mechanism takes place, dimension that is a measure of the complexity and nonlinear dynamics of the system, dependent on the diffusion order.

This theory offers new alternatives for the theoretical study of drug release process (on large time scale) in the presence of all phenomena and considering a highly complex and implicitly, non linear system. Consequently, the concentration field has cnoidal oscillation modes, generated by similar trajectories of drug particles. This means that the drug particle ensemble (at time large scale) works in a network of non linear oscillators, with oscillations around release boundary. Moreover, the normalized concentration field simultaneously depends on normalized time non linear system (through s parameter).

\section{Author details}

M. Agop ${ }^{1}$, C.Gh. Buzea ${ }^{2}$, S. Bacaita ${ }^{1}$, A. Stroe ${ }^{3}$ and M. Popa ${ }^{4}$

1 Department of Physics, Faculty of Machine Manufacturing and Industrial Management, "Gheorghe Asachi" Technical University of Iasi, Iasi, Romania

2 National Institute of Research and Development for Technical Physics, Romania

3 National College “Nicolae Balcescu”, Al. I. Cuza Bvd., Braila, Romania

4 Department of Natural and Synthetic Polymers, Faculty of Chemical Engineering and Environmental Protection, "Gheorghe Asachi" Technical University of Iasi, Iasi, Romania 


\section{References}

[1] L. Nottale, Fractal Space-Time and Microphysics: Towards a Theory of Scale Relativity, World Scientific Publishing, Singapore, 1993.

[2] L. Nottale, Scale Relativity and Fractal Space-Time - A New Approach to Unifying Relativity and Quantum Mechanics, Imperial College Press, London, 2011.

[3] L. Nottale, Fractals and the quantum theory of space time, Int. J. Mod. Phys. A, vol. 4, no. 19 , pp. 5047-5117, 1989.

[4] L. D. Landau, E. M. Lifshitz, Fluid Mechanics, $2^{\text {nd }}$ Edition, Butterworth Heinemann Publishing, Oxford, 1987.

[5] B.B. Mandelbrot, The Fractal Geometry of Nature, Freeman, San Francisco, USA, 1983.

[6] J. F. Gouyet, Physique et Structures Fractals, Masson, Paris, 1992.

[7] M.S. El Naschie, O. E. Rössler, I. Prigogine, Quantum Mechanics, Diffusion and Chaotic Fractals, Elsevier, Oxford, 1995.

[8] P. Weibel, G. Ord, O. E. Rösler, Space Time Physics and Fractality, Springer Dordrecht, 2005.

[9] M. Agop, N. Forna, I. Casian Botez, C. Bejenariu, New theoretical approach of the physical processes in nanostructures, J. Comput. Theor. Nanosci., vol. 5, no. 4, pp. 483-489, 2008.

[10] I. Casian-Botez, M. Agop, P. Nica, V. Paun, G.V. Munceleanu, Conductive and convective types behaviors at nano-time scales, J. Comput. Theor. Nanosci., vol. 7, no. 11, pp. 2271-2280, 2010.

[11] L. Nottale, Scale-relativity and quantization of the universe. I. Theoretical framework, Astron. Astrophys., vol. 327, no. 3, pp. 867-889, 1997.

[12] P.D.McCormack, L. Crane, Physical Fluid Mechanics, Academic Press, LondonNewYork, 1973.

[13] M. Agop, P.D.Ioannou, C.Gh.Buzea, P.Nica, Hydrodynamic formulation of scale relativity theory and unified superconductivity by means of a fractal string, Physica C, vol. 390, no. 1, pp. 37-55, 2003.

[14] M. Agop, P.E. Nica, P.D. Ioannou, A. Antici, V.P. Paun, Fractal model of the atom and some properties of the matter through an extended model of scale relativity, The European Phys. J. D, vol. 49, no. 2, pp. 239-248, 2008.

[15] V. P. Maslov, M. V.Fedoriuk, Semiclassical Approximation in Quantum Mechanics, Reidel, Dordrecht, 1981.

[16] W. Nolting, Quantenmechanik-Methoden und Anwendungen., Grundkurs Theoretische Physik Springer, 2004. 
[17] C. S. Park, M. G. Jeong, S.-K. Yoo, D.K. Park, arXiv:hep-th/9808137v1, 1998.

[18] R. Landauer, Irreversibility and Heat Generation in the Computing Process, IBM J. Res. Dev., vol. 5, no. 3, pp. 183-192, 1961.

[19] S. Lloyd, Use of mutual information to decrease entropy: Implications for the second law of thermodynamics, Phys. Rev. A, vol. 39, no. 10, pp. 5378-5386, 1989.

[20] C.H. Bennett, The thermodynamics of computation-a review, Int. J. Theor. Phys., vol. 21, no. 12, pp. 905-940, 1982.

[21] E. Fredkin, T. Toffoli, Conservative logic, Int. J. Theor. Phys., vol. 21, no. 3-4, pp. 219-253, 1982.

[22] W.H. Zurek, Algorithmic randomness and physical entropy, Phys. Rev. A, vol. 40, no. 8, pp. 4731-4751, 1989.

[23] C.R. Calidonna, A. Naddeo, Towards reversibility in a JJL qubit qualitative model by means of CAN2 paradigm, Phys. Lett. A, vol. 358, no. 5-6, pp. 463-469, 2006.

[24] R. Alicki, M. Horodecki, P. Horodecki, R. Horodecki, Thermodynamics of Quantum Information Systems - Hamiltonian Description, Open Sys. \& Information Dyn., vol. 11, no. 3, pp. 205-217, 2004.

[25] V. S. Popov, B. M. Karnakov, V. D. Mur, On matching conditions in the WKB method, Phys. Lett. A, vol. 210, no. 6, pp. 402-408, 1996.

[26] C. Eltschka, H. Friedrich, M. J. Moritz, J. Trost, Tunneling near the base of a barrier, Phys. Rev. A, vol. 58, no. 2, pp. 856-861, 1998.

[27] M. J. Moritz, Tunneling and reflection of long waves, Phys. Rev. A, vol. 60, no.2, pp. 832-841, 1999.

[28] S.K. Lamoreaux, Demonstration of the Casimir force in the 0.6 to $6 \mu \mathrm{m}$ range, Phys. Rev. Lett., vol. 78, no. 1, pp. 5-8, 1997.

[29] M. Bordag, U. Mohideen, V.M. Mostepanenko, New developments in the Casimir effect, Phys. Rep., vol. 353, no. 1-3, pp. 1-206, 2001.

[30] K. Milton, The Casimir effect: recent controversies and progress, J. Phys. A, vol. 37, no. 38, pp. R209-R277, 2004.

[31] V.V. Nesterenko, G. Lambiase, G. Scarpetta, Calculation of the Casimir energy at zero and finite temperature: Some recent results, Riv. Nuovo Cimento, vol. 027, no. 06, pp. 1-74, 2004.

[32] S.K. Lamoreaux, The Casimir force: background, experiments and applications, Rep. Prog. Phys., vol. 68, no. 1, pp. 201-236, 2005.

[33] G. Barton, Perturbative Casimir energies of dispersive spheres, cubes and cylinders, J. Phys. A: Math. Gen., vol. 34, no., pp. 4083-4114, 2001. 
[34] K. A. Milton, The Casimir Effect: Physical Manifestations of Zero-Point Energy (chapter 2), World Scientific, 2001.

[35] L. E. Ballentine, Quantum Mechanics (chapter 19), Prentice-Hall, 1990.

[36] C. Itzykson, J. B. Zuber, Quantum Field Theory (chapter 3), McGraw-Hill, 1985.

[37] K. Huang, Quantum Field Theory (chapter 5), John Wiley, 1998.

[38] M. Ignat, N. Rezlescu, C.Gh.Buzea, C. Buzea, About the pair breaking-time in superconductors, Phys. Lett. A vol. 195, no. 2, pp. 181-183, 1994.

[39] C. Gh. Buzea, M. Agop, N. Rezlescu, C. Buzea, T. Horgos, V. Bahrin, The Time of Diffusion and Infinite Conductivity of High-Tc Superconductors, Phys. Stat. Sol.(b), vol. 205, no. 2, pp. 595-602, 1998.

[40] M. Agop, C. Gh. Buzea, N. Rezlescu, C. Buzea, C. Marin, Wave guide perturbative solutions for the Ginzburg-Landau equation.: Infinite conductivity and discrete values of the critical temperature in superconductors, Physica C, vol.313, no. 3-4, pp. 219-224, 1999.

[41] F. Bowman, Introduction to elliptic functions with applications, English University Press London, 1961.

[42] O. Mayer, Special issues in the theory of the functions with one complex variable, vol. II, Academic Press Bucharest, 1990.

[43] M. Agop, V. Griga, C. Buzea, C. Stan, D. Tatomir, The uncertainty relation for an assembly of Planck-type oscillators. A possible GR-quantum mechanics connection, Chaos, Solitons \& Fractals, vol. 8, no. 5, pp. 809-821, 1997.

[44] S. Titeica, Quantum Mechanics, Academic Press Bucharest, 1984.

[45] M. Agop, N. Rezlescu, G. Kalogirou, Nonlinear Phenomena in Materials Science, Graphics Art Publishing House Athens, 1999.

[46] G. Burns, High-Temperature Superconductivity, Academic Press San Diego, 1992.

[47] M. Bordag, G. L. Klimchitskaya, U. Mohideen, V. M. Mostepanenko, Advances in the Casimir Effect., Oxford Univ. Press, 2009.

[48] M. Agop, N. Forna, I. Casian Botez, C. Bejenariu, New theoretical approach of the physical processes in nanostructures, J. Comput. Theor. Nanosci., vol. 5, no. 4, pp. 483-489, 2008.

[49] I. Casian-Botez, M. Agop, P. Nica, V. Paun, G.V. Munceleanu, Conductive and convective types behaviors at nano-time scales, J. Comput. Theor. Nanosci., vol. 7, no. 11, pp. 2271-2280, 2010.

[50] L. Jude, Mathematics physics equations. Theory and applications, Matrix Rom Publishing Bucharest, 2010. 
[51] K. B. Oldham, J. Spanier, The Fractional Calculus: Theory and Applications of Differential and Integration to Arbitrary Order, Dover Publications New York, 2006.

[52] A. A. Kilbas, H. M. Srivastava, J. J. Trujilto, Theory and Applications of Fractional Differential Equations, Elsevier Armsterdam, 2006.

[53] S. Bacaita, C. Uritu, M.Popa, A. Uliniuc, C. Peptu, M. Agop, Drug release kinetics from polymer matrix through the fractal approximation of motion, Smart Materials Research, article ID 264609, doi:10.1155/2012/264609, 2012.

[54] A. J. Lichtenberg, Phase-Space Dynamics of Particle, John Wiley and Sons Inc. New York, 1969.

[55] S. Popescu, Actual issues in the physics of self-structured systems, Tehnopress Publishing, Iasi, Romania, 2003.

[56] M. Toda, Theory of Nonlinear Lattices, Springer, Berlin, 1989.

[57] S. Bacaita et. al., Nonlinearities in Drug Release Process from Polymeric Microparticles: Long-Time-Scale Behaviour, Journal of Applied Mathematics, vol. 2012, article ID 653720, 2012, doi:10.1155/2012/653720. 
